\title{
Long noncoding RNA MEG3 blocks telomerase activity in human liver cancer stem cells epigenetically
}

\section{Xiaoxue Jiang}

Tongji University

Liyan wang

Tongji University

sijie xie

Tongji University

Yingjie Chen

Tongji University

Shuting Song

Tongji University

\section{Yanan Lu}

Tongji University

Dongdong Lu ( $\square$ ludongdong@tongji.edu.cn )

Tongji University https://orcid.org/0000-0002-7417-4172

\section{Research}

Keywords: liver cancer stem cell, long noncoding RNA, MEG3, P53, RERT, Telomere

Posted Date: July 16th, 2020

DOI: https://doi.org/10.21203/rs.3.rs-42251/v1

License: (c) (i) This work is licensed under a Creative Commons Attribution 4.0 International License. Read Full License

Version of Record: A version of this preprint was published at Stem Cell Research \& Therapy on November 30th, 2020. See the published version at https://doi.org/10.1186/s13287-020-02036-4. 


\section{Abstract}

Background: MEG3 is abnormally down-regulated in most tumors and inhibits tumorigenesis.

Methods: Gene infection, Western blotting and tumorigenesis test in vitro and in vivo were performed to analyze the signaling pathway.

Results: MEG3 increased the loading of P300 and RNA polymerase II onto the promoter regions of P53. Notably, MEG3 increased the methylation of histone $\mathrm{H} 3$ at lysine 27 through increasing the interplay between PRC2 and histone H3. Furthermore, MEG3 inhibited the expression of TERT by increasing the H3K27me3 and decreasing the loading of RNA pol $₫$ in TERT promoter regions. Moreover, MEG3 inhibit the activity of telomerase by reducing the binding of TERT to TERC competitively. In addition, MEG3 also increased the TERRA through reducing DNA methyltransferase DNMT3b binding to the promoter regions of TERRA competitively. Therefore, the interaction between TERC and TERT was competitively attenuated by increasing the interaction between TERRA and TERT, which inhibited the activity of telomerase in hLCSCs.In particular, MEG3 shortened the length of telomere by blocking the formation of complex maintaining telomere length(POT1-Exo1-TRF2-SNM1B) and decreasing the binding of the complex to telomere competitively, which was caused by increasing the interplay between P53 and HULC in hLCSCs.Strikingly, MEG3 inhibited the growth in vitro and in vivo of hLCSCs by reducing the activity of telomerase and attenuating telomeric repeat binding factor 2(TRF2).

Conclusions: our results demonstrated MEG3 inhibits the occurrence of human liver cancer and these findings provide an important insight into the prevention and treatment of human liver cancer.

\section{Introductions}

It has been found that human stem cells can differentiate into malignant tumor stem cells in a certain microenvironment (1). Although most of the current researches supports the tumor originating from stem cell malignant transformation, the source of cancer stem cells is still controversial, such as the inhibition of differentiation from normal stem cells or the dedifferentiation of mature cells $(2,3)$. At present, extensive research has been conducted on the mechanism of driving stem cell deterioration, such as METTL3-elF3h-mediated mRNA circulation promotes stem cell deterioration (4); FXR regulates the proliferation of small intestinal cancer stem cells (5); interleukin 22 protects intestinal stem cell resistance genotoxicity (6); C-Myc enhancer promotes the proliferative capacity of leukemia stem cells (7). Studies have shown that cancer stem cells are a subpopulation of tumors that are self-renewing and can differentiate into new tumor cells. Studies have confirmed that there are liver cancer stem cells in human liver cancer tissues, which have stem cell characteristics such as self-renewal and differentiation, and are closely related to liver cancer recurrence, metastasis, drug sensitivity and the like (8). At present, it is not clear what causes the accumulation of stem cell genetic errors, chromatin programming, chromosome instability and telomere function, and eventually evolve into cancer stem cells. 
Telomeres are a special structure of eukaryotic chromosome ends consisting of many simple repeats and related proteins rich in guanine, which have the effect of maintaining the structural integrity of chromosomes and solving the problem of end replication (9). Telomere-associated protein complexes include TRF1, TRF2, Rap1, POT1, TIN2, and TPP1 (10). They play different roles at the telomere. For example, TRF1 regulates the replication of telomeric DNA; TRF2 is involved in the formation and maintenance of the T-loop and inhibits ATM-mediated DNA damage responses and the occurrence of non-homologous end recombination (11). Telomerase is a complex of RNA and proteosome. It is a specific RNA-dependent reverse transcriptase. The telomerase core components include telomerase reverse transcriptase (TERT) and telomerase RNA (TERC). TERT is a catalytic subunit, and TERC is an RNA template during telomere extension $(12,13)$. Increased telomerase activity is associated with increased copy number of the telomerase component members TERC and TERT genes (14). Moreover, telomerase is involved in stem cell self-renewal (15). Abnormally activated telomerase in terminal tumor cells maintains telomere length to a specific length, allowing tumor cells to undergo continuous division and loss of tumor growth. Given the important role of telomere length in the malignant proliferation of tumor cells, the researchers propose to treat cancer by inhibiting the elongation of telomeres (16). From stem cells to tumor cells, telomerase contributes to the survival and reproduction of cells. Therefore, telomerase has a close relationship with cell proliferation and differentiation, and has become one of the hotspots of current cancer research. Studies have shown that telomeres in mammalian cells exhibit high levels of histone H3K9me3 and H4K2Ome3 modifications (17), and that telomeres can be transcribed by RNA polymerase II to generate long-chain non-coding RNA TERRA (Telomeric Repeat-Containing RNA) $(18,19)$. It was found that knocking out TERRA in cells resulted in a decrease in the apparent modification of $\mathrm{H} 3 \mathrm{~K} 9 \mathrm{me} 3$ at the telomere, indicating that TERRA is involved in the formation of heterochromatin at the end of the chromosome (20). In addition, the 5'-UUAGGG-3' repeat of TERRA can bind to TERC through the principle of base-complementary pairing, competitively inhibiting telomerase activity (21). However, studies have found that nuclear heterogeneous ribonucleoprotein A1 (hnRNPA1) binds to TERRA, which blocks the binding of TERRA to telomerase and activates telomerase (22). Thus, the relative balance between TERRA and hnRNPA1 determines the activity of telomerase, which in turn affects the fate of cells. In recent years, some new phenomena have been discovered to regulate telomere function in cells, but the exact mechanism is unknown. Selective extension of telomeres by DNA break-induced replication mechanisms (23-25). Telomere necrosis activates autophagic death (26) and telomeres have certain epigenetic characteristics (27). Studies have found that in vigorously dividing cells, telomerase is recruited to telomeres in the $S$ phase of the cell cycle, but only a partial understanding of the molecular mechanism of this process. Telomerase is recruited to the telomere, which is driven by the rapid interaction of telomerase and the direct interaction of chromosome ends, so inhibition of telomerase attachment to telomeres in cancer cells is a treatment strategy for disease Human telomeres have certain apparent characteristics (28).

Long non-coding RNA is a hotspot in life science research in recent years and has been widely studied. The maternal imprinted gene, MEG3, located in human chromosome 14q32.3 is a long-chain non-coding RNA that is involved in the regulation of various growth and development processes in organisms (29). 
For example, MEG3 silencing can induce mouse pluripotent stem cells (30); MEG3 inhibits activation of liver satellite cells (31); MEG3 acts as a ceRNA to regulate hepatic fat metabolism (32). Studies have shown that the expression patterns of various transcriptional variants of MEG3 are tissue-specific (33). The study found that MEG3 plays an important role in the normal growth and development of organisms. In addition, MEG3 is also involved in different pathological processes in organisms, and MEG3 is found to regulate the function of mouse insulin B cells by affecting insulin production and apoptosis. Current research indicates that MEG3 is involved in tumorigenesis (34-37). In addition, MEG3 was also found to inhibit prostate cancer progression (38) and breast cancer progression by activating NF-KB and p53 (39); MEG3 inhibits progression of osteosarcoma (40). But so far, the mechanism of action of MEG3 in tumorigenesis is still unclear, especially the regulation of stem cells is rarely reported.

In this study, the human suppressor gene P53 was found to interact with MEG3, which promoted chromatin remodeling and led to changes in telomere function in human stem cells. Under normal conditions, the amount of P53 expression in cells is maintained at a low level. However, cellular stress stimulates an increase in P53 expression (41-45). This study involved heterochromatin 1 (HP1), which is highly conserved in eukaryotes. Initially, HP1 was thought to bind to interstitial Heterochromatin (PCH)mediated gene silencing, and subsequent studies have found it involved in many other biological processes, (46-49). Numerous studies have shown that HP1a is involved in the regulation of epigenetic modifications of cancer-associated genes, which in turn affects tumor development. HP1 also protects embryonic stem cells (50-53). Numerous studies have shown that abnormalities in the methylation status of members of the PRC2 complex and histone H3K27 are closely related to tumorigenesis. For example, EZH2, EED, and SUZ12 are ubiquitously expressed in rectal cancer cells, and their expression is positively correlated with tumor malignancy and poor prognosis (54).

In this study, MEG3 inhibits human liver cancer stem cells and is involved in epigenetic regulation of histones and nucleic acids, telomere lifespan. The decrease of telomerase activity and telomere stability is an important reason for MEG3 to inhibit the growth of human liver cancer stem cells.

\section{Methods}

Tissue samples Ninety-eight cases of human primary liver cancer tissues used for analysis were obtained

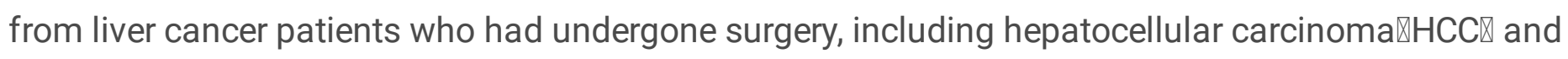

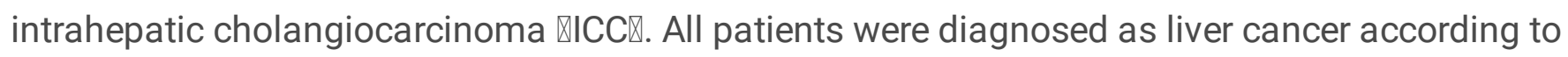
histological examination by at least three pathologists or clinicians.

\section{Human liver cancer stem cell line (hLCSC) sorting CD133/CD44/CD24/EpCAM MicroBead Kits were} purchased from Miltenyi technic(Boston,USA) and MACS ${ }^{\circledR}$ Technology operation according to and the operation according to the manufacturer.

Cell Lines , Lentivirus and Plasmids Human liver cancer stem cell line (hLCSC) was maintained in Dulbecco's modified Eagle medium(Gibco BRL Life Technologies) or Minimum Essential Medium(MEM) (Gibco BRL Life Technologies) supplemented with $10 \%$ heat-inactivated $\left(56^{\circ} \mathrm{C}, 30\right.$ minutes) fetal bovine 
serum (sigma) in a humidified atmosphere of $5 \% \mathrm{CO}_{2}$ incubator at $37^{\circ} \mathrm{C}$. rLV, rLV-Cas9 were purchased from Wu Han viraltherapy Technologies Co. Ltd. pCMV6-A-GFP, pGFP-V-RS and pMirTarget-3'UTR pMirTarget-3'UTR were purchased from Origene (Rockville, MD,USA). pCMV6-A-GFP-MEG3, pGFP-V-RS-CMEG3, pGFP-V-RS-TERT and pMartarget-3'UTR-C-TERT promoter were cloned by ourselves.

Cell infection and transfection. Cells were infected with Lentivirus and transfected with DNA plasmids using transfast transfection reagent lipofectamine ${ }^{R} 2000$ (Invitrogen) according to manufacturer's instructions.

RT-PCR Total RNA was purified using Trizol (Invitrogen) according to manufacturer's instructions. cDNA was prepared by SuperScript First-Strand Synthesis System (Invitrogen). PCR analysis was performed according to manufacturer's instructions. $\beta$-actin was used as an internal control.

Co-immunoprecipitation(IP) Cells were lysed in $1 \mathrm{ml}$ of the whole-cell extract buffer $\mathrm{A}(50 \mathrm{mM}$ pH7.6 Tris$\mathrm{HCl}, 150 \mathrm{mMNaCl}, 1 \% \mathrm{NP} 40,0.1 \mathrm{mMEDTA}, 1.0 \mathrm{mM}$ DTT,0.2mMPMSF, 0.1mM Pepstatine,0.1mM Leupeptine, $0.1 \mathrm{mM}$ Aproine). Five-hundred-microliter cell lysates was used in immunoprecipitation with antibody. Western blot was performed with a another related antibody indicated in Western blotting according to the manufacturer's instructions.

Chromatin immunoprecipitation (CHIP) assay Cells were cross-linked with $1 \%(\mathrm{v} / \mathrm{v})$ formaldehyde (Sigma) for $10 \mathrm{~min}$ at room temperature and stopped with $125 \mathrm{~mm}$ glycine for $5 \mathrm{~min}$. Crossed-linked cells were washed with phosphate-buffered saline, resuspended in lysis buffer, and sonicated for $8 \mathrm{~min}$. Chromatin extracts were pre-cleared with Protein-A/G-Sepharose beads, and immunoprecipitated with specific antibody on Protein-A/G-Sepharose beads. After washing, elution and de-cross-linking, the ChIP DNA was detected by PCR according to the manufacturer's instructions.

Chromosome conformation capture (3C)-chromatin immunoprecipitation (ChIP) Chromatin bound to the antibody-Protein-A/G-Sepharose beads were resuspended and the ChIP-3C material was detected for long range interaction with specific primers according to the manufacturer's instructions.

Super-EMSA(Gel-shift) Cells were washed and scraped in ice-cold PBS to prepare nuclei for electrophoretic gel mobility shift assay with the use of the gel shift assay system modified according to the manufacturer's instructions (Promega).

Cells proliferation CCK8 Assay The cell proliferation reagent CCK8 is purchased from Roch and the operation according to the manufacturer instruction.

Colony-Formation Efficiency Assay $10^{3}$ cells were plated on a $10 \mathrm{~cm}$ dish, the $10 \mathrm{ml}$ DMEM containing $10 \%$ FBS was added into each $10 \mathrm{~cm}$ dish of the three replicate. Cell colonies on the dishes were stained with $1 \mathrm{ml}$ of $0.5 \%$ Crystal Violet (Henan Tianfu Chemical Co.,Ltd.) according to the manufacturer's instructions. 
Xenograft transplantation in vivoThe male athymic Balb/C mice per group were injected with liver cancer stem cells at the armpit area subcutaneously. The mice were observed over 4 weeks, and then sacrificed to recover the tumors. The use of mice for this work was reviewed and approved by the institutional animal care and use committee in accordance with China national institutes of health guidelines.

\section{Results}

\section{MEG3 is down-regulated expression in human liver cancer tissues}

To validate the expression of MEG3 in human primary liver cancer, we performed hybridization In situ (ISH) and RT-PCR to detect MEG3 in the 98 cases of liver cancer patients, including liver cancer tissues and their paired adjacent noncancerous tissues from the same patient. The ISH results showed the expression of MEG3 was significantly reduced in liver cancer tissues compared to their paired adjacent noncancerous tissues $(n=62, P<0.01)$ (Figure1Aa,b\&c). The real-time RT-PCR and regular RT-PCR results showed the expression of MEG3 was significantly reduced in hepatocarocinoma tissues compared to their paired adjacent noncancerous tissues( $n=36, P<0.01)$ (Figure1B\&Ca\&b). Furthermore, we also detected the expression of miR122(FigureS1), PKM2 (FigureS2), ß-catenin (FigureS3), PTEN (FigureS4), P53 (FigureS5), TERT (FigureS6) in the 98 cases of liver cancer patients. The expression of MEG3 was positively associated with the expression of miR122, PTEN and P53, and negatively associated with the expression of PKM2, $\beta$-catenin and TERT in these liver cancer patients. Moreover, the expression level of MEG3 was closely associated with the clinic-pathological features of liver cancer patients (Supplemental Table1\&2). Collectively, these observations suggest MEG3 was downregulated in human liver cancer.

\section{MEG3 inhibits the growth of human liver cancer stem cells (hLCSCs) in vitro and in vivo}

First, human liver cancer stem cells were isolated from Huh7 cells using CD133/CD44/CD24/EpCAM microbeads (Fig. S7A). CD133, CD44, CD24, and EpCAM are expressed in human liver cancer stem cells (hLCSCs), and not in non-hepatoma stem cells (non-hLCSCs) (Fig. S7B-D). The four plasmids (pCMV6-AGFP-MEG3,pCMV6-A-GF,pGFP-V-RS-MEG3 and pGFP-V-RS) were transfected into hLCSCs, respectively, and positive cells were picked and expanded. green was expressed in these four stable cell lines (Fig. 2A). MEG3 or cyclic MEG3 was significantly increased in pCMV6-A-GFP-MEG3 group compared to the pCMV6A-GFP group and reduced in PGFP-V-RS-MEG3 group compared to the pGFP-V-RS group (Fig. 2B-E).The growth ablity was significantly reduced in pCMV6-A-GFP-MEG3 group compared to the pCMV6-A-GFP group and increased in pGFP-V-RS-MEG3 group compared to the pGFP-V-RS group $(P<0.01)$ (Fig. 2F). The Brdu positive rate was significantly reduced in PCMV6-A-GFP-MEG3 group compared to the pCMV6-A-GFP group $(42.95 \pm 3.31 \%$ vs $19.66 \pm 1.98 \%, P=0.0072145<0.01)$ and increased in pGFP-V-RS-MEG3 group compared to the pGFP-V-RS group $(38.53 \pm 2.503 \%$ vs $64.59 \pm 7.02 \%, P=0.0087726<0.01)$ (Fig. $2 \mathrm{G}$ a \& b). The colony formation ability was significantly reduced in PCMV6-A-GFP-MEG3 group compared to the pCMV6-A-GFP group $(24.62 \pm 3.08 \%$ vs $15.23 \pm 1.87 \%, P=0.007906<0.01)$ and increased in pGFP-V-RSMEG3 group compared to the pGFP-V-RS group $(22.9 \pm 2.47 \%$ vs $48.44 \pm 5.30 \%, P=0.0005529<0.01)$ (Fig. 2H a\&b). The sphere formation ability was significantly reduced in pCMV6-A-GFP-MEG3 group 
compared to the pCMV6-A-GFP group $(14.13 \pm 2.42 \%$ vs $5.37 \pm 0.73 \%, P=0.00906<0.01)$ and increased in pGFP-V-RS-MEG3 group compared to the pGFP-V-RS group $(16.13 \pm 3.26 \%$ vs $30.72 \pm 4.34 \%, P=$ $0.00216<0.01$ ) (Fig. 2l). The weight of transplanted tumors was significantly reduced in pCMV6-A-GFPMEG3 group compared with the pCMV6-A-GFP group $(0.51 \pm 0.044 \mathrm{~g}$ vs $0.173 \pm 0.025 \mathrm{~g}, \mathrm{P}=0.0000186$ $<0.01)$ and increased in pGFP-V-RS-MEG3 group compared to the pGFP-V-RS group $(0.465 \pm 0.065 \mathrm{~g}$ vs $0.96 \pm 0.126 \mathrm{~g}, \mathrm{P}=0.000000579<0.01$ ) (Fig. 2J\&2K). The appearance time of transplanted tumors in nude mice was significantly increased in PCMV6-A-GFP-MEG3 group compared with the pCMV6-A-GFP group ( $7.16 \pm 1.72$ days vs $15.0 \pm 2.09$ days, $P=0.000000076<0.01)$ and decreased in pGFP-V-RS-MEG3 group compared to the pGFP-V-RS group $(7.50 \pm 1.04$ days vs $6.0 \pm 0.89$ days, $P=0.0086<0.01)$ (Fig. 2L). The poorly differentiated was significantly decreased in pCMV6-A-GFP-MEG3 group compared with the pCMV6-A-GFP group and increased in PGFP-V-RS-MEG3 group compared to the pGFP-V-RS group (Fig. 2M).The PCNA positive rate in transplanted tumors was significantly decreased in pCMV6-A-GFPMEG3 group compared with the pCMV6-A-GFP group ( $40.65 \pm 2.88 \%$ vs $15.19 \pm 3.26 \%, P=0.0000023<0.01$ ) and increased in pGFP-V-RS-MEG3 group compared to the pGFP-V-RS group (43.64 $\pm 8.02 \%$ vs $72.88 \pm 12.71 \%, p=0.004559<0.01$ [ [Fig. $2 \mathrm{M} \& 2 \mathrm{~N}(\mathrm{a})$ ]. The Ki67 positive rate in transplanted tumors was significantly decreased in PCMV6-A-GFP-MEG3 group compared with the pCMV6-A-GFP group $(30.48 \pm 2.76 \%$ vs $12.09 \pm 3.07 \%, P=0.000099<0.01)$ and increased in pGFP-V-RS-MEG3 group compared to the pGFP-V-RS group $(27.73 \pm 4.69 \%$ vs $44.33 \pm 7.24 \%, P=0.00079<0.01)$ [Fig. 2 M\&2N(b)]. Collectively, these observations suggest that MEG3 inhibit the growth human liver cancer stem cells in vitro and in vivo.

\section{MEG3 enhances the expression of P53 in human liver cancer stem cells}

To analyze whether MEG3 affects the expression of P53 in liver cancer stem cells, we cross-linked the cells with formaldehyde and then analyzed by chromatin immunoprecipitation (CHIP) with anti-P300 and anti-RNAPollI. The results showed that the binding ability of P300 and RNAPolll to P53 promoter were significantly enhanced in the pCMV6-A-GFP-MEG3 group compared with the pCMV6-A-GFP group and reduced in the pGFP-V-RS-MEG3 compared with the pGFP-V-RS group(Fig.3A). Simultaneously, we used anti-P300, anti-RNAPolll for chromatin immunoprecipitation (CHIP)-3C analysis. The results showed that the binding capacity of P300 and RNAPolll to P53 promoter-enhancer loops (DNA LOOP) were significantly enhanced in the PCMV6-A-GFP-MEG3 group compared with the pCMV6-A-GFP group and reduced in the pGFP-V-RS-MEG3 compared with the pGFP-V-RS group (Fig. 3B). The activity of pEZX-MTP53-promoter-Luc reporter gene were significantly enhanced in the pCMV6-A-GFP-MEG3 group compared with the pCMV6-A-GFP group $(9753.68 \pm 930.63$ vs $88000.67 \pm 16199.96, p=0.0062527<0.01)$ and reduced in the pGFP-V-RS-MEG3 compared with the pGFP-V-RS group ( $9404.57 \pm 1457.29$ vs $2740.33 \pm 592.42$, $p=0.0086264<0.01$ ) (Fig. 3C). The expression of P53 in the pCMV6-A-GFP-MEG3 group compared with the pCMV6-A-GFP group and reduced in the pGFP-V-RS-MEG3 compared with the pGFP-V-RS group (Fig. 3D-F). Furthermore, the expression of P53 was slightly increased in the pCMV6-A-GFP+pcDNA3-HP1a group and decreased in the pCMV6-A-GFP+pGFP-V-RS-HP1a group compared with pCMV6-A-GFP, and expression of P53 was significantly increased in the pGFP-V-RS-MEG3+pcDNA3-HP1a group and decreased in the pCMV6-A-GFP-MEG3+pGFP-V-RS-HP1a group compared with the CMV6-A-GFP-MEG3 
group (Figure 3G). Collectively, these observations suggest MEG3 enhances the expression of P53 dependent on HP1a in human liver cancer stem cells.

\section{MEG3 promotes methylation of histone H3 at the lysine $27^{\text {th }}$ mediated by P53 in human liver cancer stem cells}

HP1 a plays a positive regulatory role in the interaction of MEG3 and P53 in liver cancer stem cells, so we sought to investigate whether MEG3 affects the methylation of histone $\mathrm{H} 3$ at lysine 27 dependent on HP1a. The interaction between MEG3 and EZH2, SUZ12, EED, RbAp46/48 were increased in pCMV6-AGFP-MEG3 group and attenuated in the pCMV6-A-GFP-MEG3+ pGFP-V-RS-P53 group compared with the pCMV6-A-GFP group (Fig.3H). The interaction between Histone H3 and EZH2, SUZ12, EED, RbAp46/48 was significantly enhanced in the pCMV6-A-GFP-MEG3 group compared with the pCMV6-A-GFP and reduced in the pGFP-V-RS-MEG3 group compared to the pGFP-V-RS group (Fig. 31). Moreover, although the interaction of EZH2, SUZ12, EED, RbAp46/48 with histone H3 was increased in the pCMV6-A-GFPMEG3 group and reduced in the pGFP-V-RS-MEG3 group compared with the pCMV6-A-GFP group, the interaction of EZH2, SUZ12, EED, RbAp46/48 with histone H3 was enhanced in pCMV6-A-GFP+pcDNA3P53 group , pCMV6-A-GFP-MEG3+pcDNA3-P53 group , pGFP-V-RS-MEG3+pcDNA3-P53 group and attenuated in pCMV6-A-GFP+ pGFP-V-RS-P53 group , pCMV6-A-GFP-MEG3+pGFP-V-RS-P53 group , pGFPV-RS-MEG3+ pGFP-V-RS-P53 group, respectively (Fig.3J). Ultimately, H3K27me1,H3K27me2 and H3K27me3 was significantly increased in the pCMV6-A-GFP-MEG3 group compared to the c pCMV6-AGFP group and reduced in the pGFP-V-RS-MEG3 group compared to the pGFP-V-RS group (Fig. 3Ka\&b). Collectively,these results suggest that MEG3-dependent P53 promotes the methylation of histone $\mathrm{H} 3$ at the lysine 27 th in human liver cancer stem cells.

\section{MEG3 inhibits the expression of telomerase reverse transcriptase}

In view of the fact that MEG3 relies on P53 to promote the methylation of histone $\mathrm{H} 3$ lysine at 27, we consider whether MEG3 affects the expression and function of telomerase reverse transcriptase (TERT) in liver cancer stem cells via H3K27me3.The binding ability of H3K27me3 to the TRET promoter DNA probe was significantly increased in pCMV6-A-GFP-MEG3 group and reduced in pGFP-V-RS-MEG3 group compared to control. On the contrary, the binding ability of RNA polll to the TRET promoter DNA probe was significantly decreased in pCMV6-A-GFP-MEG3 group and increased in pGFP-V-RS-MEG3 group compared to control (Fig.4A). Super-gel migration assay showed that the binding ability of H3K27me3 to the TERT promoter was significantly increased in PCMV6-A-GFP-MEG3 group and reduced in pGFP-V-RSMEG3 group compared to control (Fig.4B). The binding of H3K27me1, H3K27me2, H3K27me3 to TERT promoter was significantly increased in pCMV6-A-GFP-MEG3 group and reduced in pGFP-V-RS-MEG3 group compared to control (Fig. 4C). The binding of CTCF ,P300 and RNA polll to the TERT promoterenhancer(DNA loop) was decreased in pCMV6-A-GFP-MEG3 group and reduced in pGFP-V-RS-MEG3 group compared to control; In contrast, the binding of H3K27me3 to the TERT promoter-enhancer(DNA loop) was increased in pCMV6-A-GFP-MEG3 group and reduced in pGFP-V-RS-MEG3 group compared to control (Fig. 4D).Moreover,TERT promoter luciferase reporter gene activity was decreased in pCMV6-A- 
GFP-MEG3 group ( $16199.33 \pm 2720.78$ vs $4495.34 \pm 11119.53, \mathrm{p}=0.007278<0.01$ )and reduced in $\mathrm{pGFP}-\mathrm{V}$ RS-MEG3 group compared to control( $14740.67 \pm 1848.91$ vs $208068.0 \pm 20781.62, p=0.00218<0.01)$

(Fig.4E). Finally,the expression of TERT was decreased in pCMV6-A-GFP-MEG3 group and increased in pGFP-V-RS-MEG3 group compared to control (Fig. 4F\&G). The binding ability of TERT to TREC RNA probe was significantly reduced in PCMV6-A-GFP-MEG3 group compared to pCMV6-A-GFP group and increased in pGFP-V-RS-MEG3 group compared to pGFP-V-RS group. In contrast, the binding ability of P53 or HP1a and to TREC RNA probe was significantly increased in pCMV6-A-GFP-MEG3 group compared to pCMV6-AGFP group and reduced in pGFP-V-RS-MEG3 group compared to pGFP-V-RS group (Fig. 4H). Although the binding ability of TERT to TREC probe was significantly reduced in pCMV6-A-GFP-MEG3 group compared to pCMV6-A-GFP group and increased in pGFP-V-RS-MEG3 group compared to pGFP-V-RS group , P53 knockdown abrogated this action of MEG3 in hLCSCs(Fig.4la\&b). The binding ability of TERT to TREC was significantly reduced in pCMV6-A-GFP-MEG3 group compared to pCMV6-A-GFP group and increased in pGFP-V-RS-MEG3 group compared to pGFP-V-RS group. In contrast, the binding ability of P53 or HP1a and to TREC was significantly increased in pCMV6-A-GFP-MEG3 group compared to pCMV6-A-GFP group and significantly reduced in pGFP-V-RS-MEG3 group compared to pGFP-V-RS group (Fig. $4 \mathrm{~J}$ ). The telomerase activity was significantly increased in pCMV6-A-GFP-MEG3 group compared to pCMV6-A-GFP group $(0.0761 \pm 0.011$ vs $0.0055 \pm 0.0013, p=0.0043<0.01)$ and significantly reduced in pGFP-V-RS-MEG3 group compared to pGFP-V-RS group $(0.0827 \pm 0.0146$ vs $0.1773 \pm 0.0296, p=0.0094<0.01)$ (Fig. 4K). Moreover, the binding ability of P53 or HP1a and to TREC was significantly increased in the pCMV6-AGFP-MEG3 group and was significantly reduced in the pGFP-V-RS-MEG3 group compared with the pCMV6-A-GFP group. The binding ability of TERT to TREC was significantly reduced in pCMV6-A-GFPMEG3 group and was increased in the pGFP-V-RS-MEG3 group compared with the pCMV6-A-GFP group. The binding ability of P53 or HP1 $a$ and to TREC probe was enhanced in pCMV6-A-GFP+pcDNA3-P53, pCMV6-A-GFP-MEG3+pcDNA3-P53, pGFP-V-RS-MEG3+pcDNA3-P53 respectively and significantly attenuated in pCMV6-A-GFP+pGFP-V-RS-P53, pCMV6-A-GFP-MEG3+ pGFP-V-RS-P53, pGFP- V-RS-MEG3+ pGFP-V-RS-P53, respectively. The binding ability of TERT to TREC was decreased in pCMV6-AGFP+pcDNA3-P53, pCMV6-A-GFP-MEG3+ pcDNA3-P53, pGFP-V-RS-MEG3+pcDNA3-P53 respectively and enhanced in pCMV6-A-GFP+ pGFP-V-RS-P53, pCMV6-A-GFP-MEG3+pGFP-V-RS-P53, pGFP-V-RS-MEG3+ pGFP-V-RS-P53, respectively (Fig. 4L\&M). Finally, the telomerase activity was significantly decreased in the group in the pCMV6-A-GFP-MEG3 group $(0.084 \pm 0.0081$ vs $0.002 \pm 0.0002 ; P=0.00158<0.01)$ and increased in the pGFP-V-RS-MEG3 group compared with the pCMV6-A-GFP group $(0.084 \pm 0.0081$ vs $0.1787 \pm 0.0105 ; P=0.000154<0.01)$. Compared with $p C M V 6-A-G F P$, telomerase activity was significantly reduced in the pCMV6-A-GFP+pcDNA3-P53 group $(0.084 \pm 0.0081$ vs $0.0033 \pm 0.00152 ; P=0.00146<0.01)$ and increased in the pCMV6-A-GFP+ pGFP-V-RS-P53 group (0.084 \pm 0.0081 vs $0.01083 \pm 0.0095 ; P=$ $0.00177<0.01)$. Compared with the pCMV6-A-GFP-MEG3 group, the telomerase activity was significantly reduced in the pCMV6-A-GFP-MEG3+pcDNA3-P53 group $(0.00203 \pm 0.0002$ vs $0.000083 \pm 0.0000058$; $P=0.00199<0.01)$ and increased in the pCMV6-A-GFP-MEG3+pGFP-V-RS-P53 group $(0.00203 \pm 0.0002$ vs $0.0706 \pm 0.005507 ; P=0.0011<0.01)$. Compared with the pGFP-V-RS-MEG3 group, the telomerase activity was significantly reduced in the pGFP-V-RS-MEG3+pcDNA3-P53 group $(0.1787 \pm 0.0105$ vs $0.085 \pm 0.0066$; $P=0.0054<0.01)$ and increased in the pGFP-V-RS-MEG3+ pGFP-V-RS-P53 group $(0.1787 \pm 0.0105$ vs 
$0.384 \pm 0.026 ; P=0.00415<0.01$ ) (Figure $4 \mathrm{~N}$ ). Moreover, The transcriptional capacity of TERRA was significantly increased in pCMV6-A-GFP-MEG3 group compared to pCMV6-A-GFP group and decreased in pGFP-V-RS-MEG3 group compared to pGFP-V-RS group (Fig.5Aa\&b, Figure5B). Although the interaction between TERRA and TERT was significantly increased in PCMV6-A-GFP-MEG3 group compared to pCMV6-A-GFP group, it was not significantly altered in pCMV6-A-GFP-MEG3+ pGFP-V-RS-P53 group compared to pCMV6-A-GFP group. Furthermore, although the interaction between TERC and TERT was significantly diminished in pCMV6-A-GFP-MEG3 group compared to pCMV6-A-GFP group, it is not significantly altered in pCMV6-A-GFP-MEG3+ pGFP-V-RS-P53 group compared to pCMV6-A-GFP group (Fig.5C\&D). RNA super-EMSA showed the interaction between TERRA and TERT was significantly increased in pCMV6-A-GFP-MEG3 group compared to pCMV6-A-GFP group and significantly decreased in pGFP-V-RS-MEG3 group compared to pGFP-V-RS group. However, P53 or HP1 a knockdown abrogated this MEG3 action (Fig. 5Ea\&b). RIP showed the interaction between TERRA and TERT was significantly increased in PCMV6-A-GFP-MEG3 group compared to pCMV6-A-GFP group and significantly decreased in pGFP-V-RS-MEG3 group compared to pGFP-V-RS group. However, P53 or HP1 a knockdown abrogated this MEG3 action. Furthermore, the interaction between TERC and TERT was significantly decreased in pCMV6-A-GFP-MEG3 group compared to pCMV6-A-GFP group and significantly increased in pGFP-V-RSMEG3 group compared to PGFP-V-RS group. However, P53 or HP1 a knockdown abrogated this MEG3 action (Fig.5F).Finally,telomerase activity was significantly diminished in pCMV6-A-GFP-MEG3 group compared to pCMV6-A-GFP group $(0.095 \pm 0.0065$ vs $0.0037 \pm 0.0015 ; P=0.0012<0.01)$. However , compared with the pCMV6-A-GFP group, telomerase activity was not significantly altered in pCMV6-AGFP-MEG3+ pGFP-V-RS-P53 (0.095 \pm 0.0065 vs $0.089 \pm 0.001 ; P=0.1704>0.05)$ and in pCMV6-A-GFPMEG3+ pGFP-V-RS-TERRA ( $0.095 \pm 0.0065$ vs $0.085 \pm 0.0047 ; P=0.00754>0.05)$ (Fig. 5G). Collectively, these results suggest that MEG3 significantly reduces telomerase activity in liver cancer stem cells by increasing TERRA dependent on P53 and HP1a.

\section{MEG3 inhibits telomere stability in human liver cancer stem cells by promoting the interaction of P53 with long-chain non-coding RNA HULC}

Given that studies have shown that POT1, Exo1, SNM1B, TRF2, and CST/AAF are important related proteins that maintain telomere length. We will analyze whether MEG3 has an effect on the effects of these proteins. The interaction between CST/AAF and POT1 was significantly increased in pCMV6-A-GFPMEG3 group compared to pCMV6-A-GFP group and decreased in pGFP-V-RS-MEG3 group compared to pGFP-V-RS group,and the interaction between TRF2 and SNM1B was significantly weakened in pCMV6-AGFP-MEG3 group compared to pCMV6-A-GFP group and increased in pGFP-V-RS-MEG3 group compared to pGFP-V-RS group(Figure6A). Moreover, the binding ability of POT1, Exol, TRF2, SNM1B to the telomeric DNA probe was significantly decreased in PCMV6-A-GFP-MEG3 group compared to pCMV6-A-GFP group and increased in pGFP-V-RS-MEG3 group compared to pGFP-V-RS group, and the binding ability of CST/AAF to the telomeric DNA probe was significantly increased in pCMV6-A-GFP-MEG3 group compared to pCMV6-A-GFP group and decreased in pGFP-V-RS-MEG3 group compared to pGFP-V-RS group(Figure6B). The binding ability of POT1, Exol, TRF2, SNM1B, HP1a to telomeric DNA was significantly reduced in pCMV6-A-GFP-MEG3 group compared to pCMV6-A-GFP group and increased in 
pGFP-V-RS-MEG3 group compared to pGFP-V-RS group,and the binding ability of CST/AAF to telomeric DNA was significantly increased in pCMV6-A-GFP-MEG3 group compared to pCMV6-A-GFP group and decreased in pGFP-V-RS-MEG3 group compared to pGFP-V-RS group(Figure6C). Furthermore, there was no significant difference in the transcription of long-chain non-coding RNA HULC among pCMV6-A-GFP group, pCMV6-A-GFP-MEG3 group, pGFP-V-RS group , pGFP-V-RS-MEG3 group (Fig. 7A). The binding ability of P53 to HULC was significantly enhanced, and the binding ability of POT1, Exol, TRF2, SNM1B and HULC was significantly reduced in PCMV6-A-GFP-MEG3 group compared to pCMV6-A-GFP group. On the contrary, the binding ability of P53 to HULC was significantly reduced, and the binding ability of POT1, Exol, TRF2, SNM1B and HULC was significantly enhanced in pGFP-V-RS-MEG3 group compared to pGFPV-RS group(Fig.7B). Compared to control group, the binding ability of P53 to HULC was significantly increased in the pCMV6-A-GFP-MEG3 group and pCMV6-A-GFP-MEG3+pCMV6-A-GFP-HULC group, and was significantly reduced in the pGFP-V-RS-MEG3 group and pGFP-V-RS-MEG3+ pGFP-V-RS-HULC group.And the binding ability of P53 to HULC was not significantly altered in pCMV6-A-GFP-MEG3+pGFPV-RS-P53 and pGFP-V-RS-MEG3+pCMV6-A-GFP-P53 compared to control group(Fig.7C). Furthermore, the binding ability of POT1, Exol, TRF2, SNM1B to telomeric DNA was significantly reduced and the binding ability of CST / AAF to telomeric DNA was significantly increased in pCMV6-A-GFP-MEG3 group compared with pCMV6-A-GFP group, and the binding ability of POT1, Exol, TRF2, SNM1B to telomeric DNA was increased and the binding ability of CST /AAF to telomeric DNA was significantly decreased in pGFP-VRS-MEG3 group compared to pGFP-V-RS group. However, the binding ability of POT1, Exol, TRF2, SNM1B, CST/AAF to telomeric DNA was not significantly altered in pCMV6-A-GFP-MEG3+pGFP-V-RS-P53 group, pCMV6- A-GFP-MEG3+ pCMV6-A-GFP-HULC group, pGFP-V-RS-MEG3+pCMV6-A-GFP-P53 group and pGFP-V-RS-MEG3+ pGFP-V-RS-HULC group (Fig. 7D). Super-EMSA results showed that the binding ability of TRF2 to telomere DNA probe was significantly reduced in pCMV6-A-GFP-MEG3 group compared with the pCMV6-AC-GFP group and increased in the pGFP-V-RS-MEG3 group compared with the pGFP-V-RS group.However, the ability of TRF2 to bind to telomeric DNA was significantly not altered in the pGFP-VRS-MEG3+pCMV6-A-GFP-P53 group and the pGFP-V-RS-MEG3+ pGFP-V-RS-HULC group (Fig.7Ea\&b). The length of telomere was significantly reduced in the pCMV6-A-GFP-MEG3 group compared with the pCMV6-A-GFP group and increased in the pGFP-V-RS-MEG3 group compared to pGFP-V-RS group(Fig. 7F\&G). In addition, the quantitative PCR analysis showed telomere length was significantly reduced in pCMV6-A-GFP-MEG3 group compared to the pCMV6-A-GFP group (1.44 \pm 0.289 Vs $0.213 \pm 0.045$; $P=0.00673<0.01)$ and increased in the pGFP-V-RS-MEG3 group compared with the pGFP-V-RS group $(1.413 \pm 0.165$ vs $3.51 \pm 0.424 ; P=0.00778<0.01)$ (Fig. 7H). Although the length of telomere was significantly reduced in the pCMV6-A-GFP-MEG3 group compared with the pCMV6-A-GFP group and increased pGFP-V-RS-MEG3 group compared to pGFP-V-RS group, it was significantly not altered in pCMV6-A-GFP-MEG3+pGFP-V-RS-P53 group, pCMV6-A-GFP-MEG3+pCMV6-A-GFP-HULC group, pGFP-VRS-MEG3+ pcDNA3-P53 group and the pGFP-V-RS-MEG3+ pGFP-V-RS-HULC group(Fig. 7l). In addition,although quantitative PCR analysis of telomere length showed telomere length was significantly reduced in the pCMV6-A-GFP-MEG3 group compared with the pCMV6-A-GFP group (1.643 \pm 0.284 VS $0.243 \pm 0.042 ; P=0.00661<0.01)$ and increased pGFP-V-RS-MEG3 group compared to pGFP-V-RS group $(1.513 \pm 0.208$ vs $4.21 \pm 0.311 ; P=0.00602<0.01)$, it was significantly not altered in pCMV6-A-GFP- 
MEG3+pGFP-V-RS-P53 group (1.643 \pm 0.284 vs $1.347 \pm 0.185 ; P=0.10788>0.05)$, pCMV6-A-GFPMEG3+pCMV6-A-GFP-HULC group (1.643 \pm 0.284 vs $1.527 \pm 0.105 ; P=0.296>0.05)$, pGFP-V-RSMEG3+pcDNA3-P53-hLCSCs group $(1.513 \pm 0.208$ vs $1.313 \pm 0.138) ; P=0.071>0.05)$ and pGFP-V-RSMEG3+pGFP-V-RS-HULC group (1.513 \pm 0.208 vs $1.446 \pm 0.197 ; P=0.178>0.05)$ compared with the pCMV6A-GFP group (Fig. 7J). Collectively, these results suggest that MEG3 significantly inhibits telomere elongation in liver cancer stem cells, dependent on both P53 and HULC.

\section{The Excessive of telomerase or TRF2abrogates the inhibitory effect of MEG3 on the growth of human liver cancer stem cells}

Telomeres play an important role in the evolution of cancer stem cells. To analyze whether MEG3 inhibits the growth of liver cancer stem cells in vitro is related to telomeres. We constructed related cell lines and then performed rescue experiments on tumorigenic ability in vitro and in vivo. Compared to pCMV6-A-GFP group, MEG3 was overexpressed in pCMV6-A-GFP-MEG3 group, pCMV6-A-GFP-MEG3+ rLV-TERT group, pCMV6-A-GFP-MEG3+pGFP-V-RS-TERT group, pCMV6-A-GFP-MEG3+ telomerase inhibitor group , and the TERT expression was decreased in pCMV6-A-GFP-MEG3 group, pCMV6-A-GFP + pGFP-V-RS-TERT group, pCMV6-A-GFP-MEG3+telomerase inhibitor group and increased in pCMV6-A-GFP-MEG3+rLV-TERT group (Figure 8A). Compared to pCMV6-A-GFP group, cell growth was inhibited in pCMV6-A-GFP-MEG3 group, pCMV6-A-GFP-MEG3 + pGFP-V-RS-TERT group, telomerase inhibitor group, pCMV6-A-GFP-

MEG3+telomerase inhibitor group $(P<0.01)$. There was no significantly sifference between pCMV6-A-GFP group and pCMV6-A-GFP-MEG3+rLV-TERT group $(P>0.05)$. Moreover, cells proliferation was slower in the pCMV6-A-GFP-MEG3+pGFP-V-RS-TERT group, pCMV6-A-GFP-MEG3+ telomerase inhibitor group than in pCMV6-A-GFP group (Figure 8B).Compared to pCMV6-A-GFP group, BrdU positive rate was significantly decreased in the pCMV6-A-GFP-MEG3 group hLCSCs $(19.08 \pm 2.96 \%$ vs $37.39 \pm 4.59 \%, P=0.0000819<0.01)$, pCMV6-A-GFP-MEG3 + pGFP-V-RS-TERT group $(10.03 \pm 1.64 \%$ vs $37.39 \pm 4.59 \%, P=0.0000186<0.01)$, telomerase inhibitor group $(20.77 \pm 7.24 \%$ vs $37.39 \pm 4.59 \%, P=0.00193<0.0193)$, pCMV6-A-GFP-

MEG3+telomerase inhibitor group $(9.32 \pm 2.29 \%$ vs $37.39 \pm 4.59 \%, P=0.0000234<0.01)$. Moreover, the positive rate of BrdU in liver cancer stem cells was lower in pCMV6-A-GFP-MEG3+pGFP-V-RS-TERT group and pCMV6-A-GFP-MEG3 + telomerase inhibitor group. However, compared with the pCMV6-A-GFP group, the BrdU positive rate of hLCSC in liver cancer stem cells was not significantly altered in the pCMV6-AGFP-MEG3 + rLV-TERT group $(35.97 \pm 5.57 \%$ vs $37.39 \pm 4.59 \%, P=0.2804>0.05$ (Fig. 8C).Compared with the pCMV6-A-GFP group, the colonfy formation rate was significantly decreased in the pCMV6-A-GFPMEG3 group hLCSCs $(18.86 \pm 3.79 \%$ vs $40.19 \pm 4.92 \%, \mathrm{P}=0.00795<0.01)$, pCMV6-A-GFP-MEG3 + pGFP-VRS-TERT group $(9.52 \pm 1.54 \%$ vs $40.19 \pm 4.92 \%, P=0.0068<0.01)$, telomerase inhibitor group $(21.19 \pm 4.52 \%$ vs $40.19 \pm 4.92 \%, P=0.028<0.05)$, pCMV6-A-GFP-MEG3+ telomerase inhibitor group $(10.97 \pm 3.07 \%$ vs $40.19 \pm 4.92 \%, P=0.000724<0.01)$. Moreover, the colony formation rate of liver cancer stem cells was lower in the pCMV6-A-GFP-MEG3 + pGFP-V-RS-TERT group and the pCMV6-A-GFP-MEG3 + telomerase inhibitor group. However, there was no significant change in colony formation rate of liver cancer stem cell between the pCMV6-A-GFP-MEG3 + rLV-TERT-hLCSCs group and pCMV6-A-GFP group $(42.13 \pm 2.77 \%$ vs $40.19 \pm 4.92 \%, P=0.336>0.05$ ) (Fig. 8D). Compared to pCMV6-A-GFP group, the sphere formation rate was significantly decreased in the pCMV6-A-GFP-MEG3 group hLCSCs $(26.69 \pm 4.55 \%$ vs $56.49 \pm 3.55 \%$, 
$P=0.008557)$, pCMV6- A-GFP-MEG3 + pGFP-V-RS-TERT group (10.33 $\pm 4.29 \%$ vs 56.49 $\pm 3.55 \%$, $\mathrm{P}=0.00127)$, telomerase inhibitor group (27.58 $\pm 2.37 \%, \mathrm{P}=0.000405), \mathrm{pCMV6}-\mathrm{A}-\mathrm{GFP}-\mathrm{MEG}+$ telomerase inhibitor group $(12.85 \pm 3.43 \%$ vs $56.49 \pm 3.55 \%, P=0.00348)$. Moreover, the sphere formation rate of liver cancer stem cells was lower in the pCMV6-A-GFP-MEG3 + pGFP-V-RS-TERT group and the pCMV6-A-GFPMEG3+ telomerase inhibitor group. However, compared with the pCMV6-A-GFP group, the sphere formation rate of liver cancer stem cell hLCSC in pCMV6-A-GFP-MEG3 + rLV-TERT -hLCSCs group did significantly not change (59.56 $\pm 5.15 \%$ vs $56.49 \pm 3.55 \%, P=0.00348)$ (Fig. 8E).

Next $\rrbracket$ We inoculated these liver cancer stem cells into the subcutaneous skin of Balb/C nude mice with low immunity (6 in each group, 36 in total). Figure 8Fa shows photographs of transplanted tumors (xenograft).Compared to pCMV6-A-GFP group, the xenografts weight was significantly reduced in the pCMV6-A-GFP-MEG3 group (0.298 $\pm 0.046 \mathrm{~g}$ vs $0.837 \pm 0.079 \mathrm{~g}, \mathrm{P}=0.000022<0.01)$, pCMV6-A-GFP-MEG3 + pGFP-V-RS-TERT group $(0.127 \pm 0.186 \mathrm{~g}$ vs $0.837 \pm 0.079 \mathrm{~g}, \mathrm{P}=0.0000014<0.01)$, telomerase inhibitor group $(0.28 \pm 0.0529 \mathrm{~g}$ vs $0.837 \pm 0.079 \mathrm{~g}, \mathrm{P}=0.00000228<0.01)$, pCMV6-A-GFP-MEG3+ telomerase inhibitor group $(0.132 \pm 0.0365 \mathrm{~g}$ vs $0.837 \pm 0.079 \mathrm{~g}, \mathrm{P}=0.00000317<0.01)$.Moreover, the transplanted tumor weight was lower in the pCMV6-A-GFP-MEG3 + pGFP-V-RS-TERT group and the pCMV6-A-GFP-MEG3+ telomerase inhibitor group. However, compared with the pCMV6-A-GFP group, there was no significant change in the transplanted tumor weight in the pCMV6-A-GFP-MEG3 + p rLV-TERT-hLCSCs group (0.892 $\pm 0.048 \mathrm{~g}$ vs $0.837 \pm 0.079 \mathrm{~g}, \mathrm{P}=0.1149>0.05$ ) (Fig. 8Fb). Compared to pCMV6-A-GFP group , the appearance time of xenograft tumor was significantly increased in the pCMV6-A-GFP-MEG3 group (12.83 \pm 1.17 days vs 7.5 \pm 1.05 days, $P=0.000246<0.01)$, pCMV6-A-GFP-MEG3 + pGFP-V-RS-TERT (17.16 \pm 1.72 days vs $7.5 \pm 1.05$ days, $\mathrm{P}=0.0000032<0.01)$, telomerase inhibitor group $(11.83 \pm 1.83$ days vs $7.5 \pm 1.05$ days, $\mathrm{P}=$ $0.0035<0.01)$ and pCMV6-A-GFP-MEG3+ telomerase inhibitor group (16.17 \pm 2.48 days vs $7.5 \pm 1.05$ days, $\mathrm{P}=0.000399<0.01)$. Moreover, the appearance time of xenografts tumor was longer in the pCMV6-A-GFPMEG3 + pGFP-V-RS-TERT group and the pCMV6-A-GFP-MEG3 + telomerase inhibitor group. However, there was no significant significance between pCMV6-A-GFP group and the pCMV6-A-GFP-MEG3 + rLVTERT-hLCSCs group (7.83 \pm 0.75 days vs $7.5 \pm 1.05$ days, $P=0.305>0.05$ )(Fig. 8F c). The transplanted tumors produced in nude mice of the six cell lines were then subjected to HE staining (Fig. 8Ga) and proliferating cell nuclear antigen (PCNA) immunohistochemical staining. Compared to pCMV6-A-GFP group , the positive rate of PCNA was significantly reduced in the pCMV6-A-GFP-MEG3 group (31.32 $\pm 7.46 \%$ vs 56.34 $\pm 5.53 \%, P=0.00057<0.01)$, pCMV6-A-GFP-MEG3 + pGFP-V-RS-TERT group $(13.81 \pm 2.91 \%$ vs $56.34 \pm 5.53 \%, P=0.00000423<0.01)$, telomerase inhibitor group $(33.32 \pm 5.61 \%$ vs $56.34 \pm 5.53 \%, P=0.00000434<0.01)$ and pCMV6-A-GFP-MEG3+ telomerase inhibitor group $(14.76 \pm 4.35 \%$ vs $56.34 \pm 5.53 \%, P=0.0000475<0.01)$. Moreover, the positive rate of PCNA was lower in the pCMV6-A-GFPMEG3+pGFP-V-RS-TERT group and the pCMV6-A-GFP-MEG3+ telomerase inhibitor group. However, there was no significant significance between pCMV6-A-GFP group and the pCMV6-A-GFP-MEG3 + rLV-TERThLCSCs group ( $59.91 \pm 5.28 \%$ vs $56.34 \pm 5.53 \%, P=0.1379>0.05$ ) (Fig. 8 Ga \& b). Collectively, thers results suggest that MEG3 can significantly inhibit the hLCSCs growth ability in vitro and in vivo dependent on telomerase. 
Furthermore, we constructed three stable cell lines, including pCMV6-A-GFP group, pCMV6-A-GFP-MEG3 group, pCMV6-A-GFP-MEG3 +rLV-TRF2group.Compared to pCMV6-A-GFP group, MEG3 was significantly increased in pCMV6-A-GFP-MEG3 group, pCMV6-A-GFP-MEG3 +rLV-TRF2group, respectively(Fig. 8H). Compared to pCMV6-A-GFP group, the expression of TRF2 was decreased in pCMV6-A-GFP-MEG3 group and increased in pCMV6-A-GFP-MEG3+rLV-TRF2 group (Fig.8H). Compared to pCMV6-A-GFP group, the growth ability was attenuated in pCMV6-A-GFP-MEG3 group $(P<0.01)$. However, there was no significant difference of growth ability between pCMV6-A-GFP group and pCMV6-A-GFP-MEG3 + rLV-TRF2-hLCSCs group (Fig. 8I). Compared to pCMV6-A-GFP group, the BrdU positive rate was decreased in pCMV6-A-GFPMEG3group $(18.11 \pm 3.12 \%$ vs $36.74 \pm 4.23 \%, P=0.00097<0.01)$. However, there was no significant difference of Brdu positive rate between pCMV6-A-GFP group and pCMV6-A-GFP-MEG3 + rLV-TRF2hLCSCs group ( $39.44 \pm 6,89 \%$ vs $36.74 \pm 4.23 \%, P=0.3542>0.05)$ (Fig. 8J). Compared to pCMV6-A-GFP group, the colony formation rate rate was decreased in pCMV6-A-GFP-MEG3 group $(11.34 \pm 2.05 \%$ vs $64.96 \pm 7.71 \%, P=0.00535<0.01)$. However, there was no significant difference in colony formation rate between pCMV6-A-GFP group and pCMV6-A-GFP-MEG3 + rLV-TRF2-hLCSCs group $(68.89 \pm 5.76 \%$ vs 64.96). $\pm 7.71 \%, P=0.3224>0.05$ ) (Fig. 8Ka\&b). Compared to pCMV6-A-GFP group, the sphere formation rate rate was decreased in PCMV6-A-GFP-MEG3 group $(11.85 \pm 3.93 \%$ vs $24.81 \pm 4.94 \%$, $\mathrm{P}=0.000528<0.01)$. However, there was no significant difference in colony formation rate between $\mathrm{pCMV6-}$ A-GFP group and pCMV6-A-GFP-MEG3 + rLV-TRF2-hLCSCs group ( $26.04 \pm 9.41 \%$ vs 24.81$) \pm 4.94 \%$, $\mathrm{P}=0171>0.05$ ) (Fig. 8L). Compared to pCMV6-A-GFP group, the xenograft tumor weight was significantly decreased in pCMV6-A-GFP-MEG3 group $(0.37 \pm 0.053 \mathrm{~g}$ vs $0.86 \pm 0.108 \mathrm{~g}, \mathrm{P}=0.000175<0.01)$. However, there was no significant difference in xenograft tumor weight between pCMV6-A-GFP group and pCMV6A-GFP-MEG3 + rLV-TRF2-hLCSCs group (0.915 $\pm 0.1559 \mathrm{~g}$ vs $0.86 \pm 0.108 \mathrm{~g}, \mathrm{P}=0.2143>0.05)$ (Fig. 8Ma\&b). Compared to pCMV6-A-GFP group, the appearance time of xenograft tumor was increased in pCMV6-AGFP-MEG3 group $(14.33 \pm 1.75$ days vs $7.83 \pm 1.17$ days, $P=0.00001124<0.01)$. However, there was no significant difference in xenograft tumor appearance time between pCMV6-A-GFP group and pCMV6-AGFP- MEG3 + rLV-TRF2- $h$ LCSCs group ( $8.33 \pm 1.37$ days vs $7.83 \pm 1.17$ days, $P=0.2448>0.05)$ (Fig. 8LC). Furthermore, HE staining and proliferating cell nuclear antigen (PCNA) immunohistochemical staining were performed. Compared to pCMV6-A-GFP group, the PCNA positive rate was significantly reduced in pCMV6-A-GFP-MEG3 group (68.84 $\pm 9.12 \%$ vs $68.84 \pm 9.12 \%, P=0.00001644<0.01)$. However, there was no

significant difference in PCNA positive rate between pCMV6-A-GFP group and pCMV6-A-GFP-MEG3+rLVTRF2-hLCSCs group (72.82 \pm 6.82 days vs $68.84 \pm 9.12 \%, P=0.18139>0.05)$ (Fig. 8Na\&b). Collectively, these results suggest that excessive MEG3 can significantly inhibit the growth ability in vitro and in vivo by inhibiting TRF2 in liver cancer stem cells.

\section{Discussion}

To date, we mainly analyzed the cellular and molecular mechanisms by which MEG3 inhibits the progression of human liver cancer stem cells. these observations indicated that MEG3-dependent P53 increased the methylation modification of histone $\mathrm{H} 3$ of the 27th lysine in the TERT promoter region, thereby inhibiting the expression of TERT.Furthermore, the excessive MEG3 reduces the binding of TERC 
to TERT , thereby further inhibiting the activity of telomerase in human liver cancer stem cells.Moreover, MEG3 relies on the interaction of P53 and HULC to decrease the stability of telomeres in human liver cancer stem cells.And further our findings demonstrated that both decreased telomerase activity and decreased telomere stabilizing protein TRF2 were important reasons for MEG3 inhibiting human liver cancer stem cell growth (Fig. 9). The discussions are as follows:

\section{MEG3 acts as a liver cancer-associated long non-coding RNA and plays an important role in inhibiting the malignant proliferation of human iver cancer stem cells}

Long non-coding RNA MEG3 is a maternal-expressed imprinting gene involved in the regulation of various growth and development processes in organisms (55-58). For example, the expression of MEG3 in bladder cancer cells is significantly reduced, activating autophagy, which in turn enhances cell proliferation and viability $(\mathbf{5 9}, \mathbf{6 0})$. Therefore, we believe that MEG3 plays an important role in inhibiting tumorigenesis.

Our results confirm that MEG3 is down-regulated in human hepatocarcinoma tissues, especially in the 98 cases of liver cancer tissues we studied. The expression of MEG3 is consistently low, and the expression rate is down-regulated by gender, alpha-fetoprotein level, hepatitis B virus infection, Edmondson's pathological grade, Hepatic function Child-Pugh classification, TNM clinical stage, liver cancer metastasis and other effects,suggesting that MEG3 may play a role in the inhibition of liver cancer through different regulatory mechanisms at different stages of liver cancer development and different microenvironment conditions, so it is reasonable to speculate that MEG3 may play an important role in determining the fate of liver cancer stem cells.

Notably, our results indicate that overexpression of MEG3 significantly inhibited the proliferation of human liver cancer stem cells in vitro and in vivo. According to research reports, excessive MEG3 can inhibit tumor growth through a variety of mechanisms. For example, in non-small cell lung cancer and gliomas, MEG3 promotes the production of apoptosis by promoting the expression of the tumor suppressor gene P53 $(\mathbf{6 1}, \mathbf{6 2})$. Also, in some cervical cancer cells, MEG3 can stop the cell cycle in the G2/M phase and cause apoptosis, thereby inhibiting tumor cell growth (63). Furthermore, in human endometrial cancer cells, MEG3 inhibits tumor cell proliferation by blocking the Notch signaling pathway. These studies indicate that MEG3 is a very important tumor suppressor gene (64). Our results are consistent with these reports. Significantly, our pevious studies found that MEG3 was significantly positively correlated with the expression of miR122, PTEN, and P53 in liver cancer tissues, and negatively correlated with the expression of PKM2, $\beta$-catenin, and TERT, suggesting that MEG3 may alter the expression and function of these genes that are closely related to the development of liver cancer by several complex signaling pathways. These results provide information for further investigation of the mechanism by which MEG3 inhibits the development of liver cancer.

A large number of studies have shown that there are liver cancer stem cells in liver cancer tissues, which have stem cell characteristics such as self-renewal and differentiation, and are closely related to the recurrence, metastasis, prognosis, sensitivity of chemical drugs and the efficacy of radiotherapy in 
human liver cancer patients (65). It has been reported that a small number of liver cancer stem cells can be isolated from some human liver cancer cell lines, such as Huh7 (67). In this study, we used isolated and identified human liver cancer stem cell hLCSCs to reveal that MEG3 can inhibit the growth of human liver ca ner stem cells through epigenetic mechanisms at the cellular and molecular levels, and that MEG3 mainly affects the telomere lifespan.

\section{MEG3-dependent P53 plays a role in the regulation of human liver cancer stem cells}

First, our study found that MEG3 promotes the expression of the tumor suppressor gene P53 in human liver cancer stem cells at the transcriptional and translational levels. The primary mechanism is that MEG3 increases the ability of RNA polymerase II to bind to the P53 promoter region. Our results show that MEG3 can form a circular structure in human live cancer stem cells, and it is likely that the circular MEG3 forms a supercoiled topology, and both RNA polymerase II and P300 are loaded into the transcriptional regulatory region of P53. Of course, there may be other indirect regulation mechanisms. Studies have shown that P53 is a multifunctional transcription factor that has been shown to be a very important tumor suppressor gene(68). In general, the expression of P53 in cells is maintained at a low level, while the expression of P53 is increased and functioning under cellular stress stimulation (69). Studies have shown that P53 can induce cell cycle arrest and promote DNA repair or induce apoptosis through multiple pathways (68). However, a large number of studies have shown that the mutation rate of P53 in human tumor cells is high, and there are deletions or mutations in more than $50 \%$ of malignant tumors, which are closely related to the occurrence and development of tumors (70). There are also studies showing that MEG3 can inhibit tumor growth by up-regulating P53 expression. For example, overexpression of MEG3 in non-small cell lung cancer and glioma cells promotes P53 expression and ultimately inhibits malignant proliferation of tumor cells $(\mathbf{6 1 , 6 2})$. This study mainly found that MEG3 promoted the expression of P53, consistent with the above report.

Secondly, according to the literature, human hepatoma cell line Hep3B does not express P53 (71), so in the previous study we found that MEG3 can inhibit the malignant proliferation of human hepatoma cell Hep3B in a P53-independent manner. In this study, our results also show that MEG3 exerts anti-cancer function through P53 in human hepatoma stem cells, mainly based on the following: (a) P53 can interact with specific regions of MEG3 in human liver cancer stem cells, and this interaction can mediate the function of MEG3 played in human liver cancer stem cells. (b) although MEG3 overexpression inhibits the growth of human hepatoma stem cells in vitro and in vivo, P53 knockdown abolishes the tumor suppressor function of MEG3, including the in vitro proliferation ability of human liver cancer stem cells, the percentage of $S$ phase cells, the ability of cell colony formation, cell sphere formation ability and tumorigenic ability in nude mice. (c) MEG3 relies on P53 to inhibit telomere lifespan in human liver cancer stem cells. These results suggest that MEG3 can inhibit the growth of human liver cancer stem cells in a P53-dependent manner. It is worth noting that our study found that MEG3 not only binds to wild-type P53, but also binds to mutant P53 (including R175H, P275S, L344P). These combinations may allow MEG3 to perform different functions and need to be further confirmed. It has been reported that the expression of P53 in tumors is usually associated with missense mutations, ie, a gene encoding P53 is mutated to 
synthesize a mutant of P53 (72). Most mutant P53 have proto-oncogene activity and can promote malignant transformation of cells. However, some mutant P53 have a pro-apoptotic ability to exert a tumor suppressor function (72). Therefore, based on our results, it can be speculated that human liver cancer stem cells isolated from Huh7 cells may express wild-type P53 or some mutant P53, and this mutant may contain R175H, P275S, L344P. Therefore, MEG3 exerts a tumor suppressor function dependent on wild-type or mutant P53 in human liver cancer stem cells, and its detailed mechanism needs further confirmation. In addition, our study found that MEG3 can affect the expression and function of telomere-related genes, such as telomerase reverse transcriptase TERT, telomerase RNA TERC, telomere repeat RNA sequence TERRA, telomere length maintenance protein POT1 Exo1, TRF2, SNM1B, CST/AAF, etc., while knockdown of P53 abolishes the effect of MEG3 on the expression and function of these genes. Therefore, we believe that MEG3 promotes the expression of P53 in human liver cancer stem cells, which in turn mediates the role of MEG3 in suppressing liver cancer. It can be seen that there is a mutual regulation between MEG3 and P53, and the presence of P53 plays an important role in the role of MEG3 in suppressing cancer.

\section{MEG3, P53 and HP1a synergistically affect the histone H3 modification in human liver cancer stem cells}

Our study not only revealed the intermodulation of MEG3 and P53, but also found that this interaction altered the epigenetic modification of histones and nucleic acids. Studies have shown that changes in epigenetic modifications (such as DNA methylation, histone methylation, histone acetylation, etc.) can cause changes in gene expression and function, which may ultimately affect cell fate (73). Our results in this study indicate that MEG3 is involved in epigenetic modification of histones in human liver cancer stem cells. The main evidences are as follows: (a) MEG3-dependent P53 increases methylation of at the 27th lysine of histone H3 (H3K27me1/2/3). (b) The function of MEG3 requires the participation of the epigenetic modification factor heterochromatin protein HP1a. (c) MEG3 regulates several long non-coding RNAs (such as TERC, TERRA, HULC) with the participation of P53 and produces new biological functions. (d) MEG3 altered the activities of histone acetyltransferase P300 and DNA methyltransferase DNMT3b, and P53 played an important role in this process. MEG3 ultimately inhibits the growth of human liver cancer stem cells through these mechanisms.

So how does MEG3 work on human liver cancer stem cells by altering histone modifications or apparent modifications of nucleic acids? And how does P53 and HP1a work synergistically with MEG3? In this study, we reveal some important ways. For example, we found that MEG3-dependent PRC2 complex increases the H3K27me3 modification of the telomerase reverse transcriptase TERT promoter region and inhibits the expression of TERT, but P53 knockdown can abolish the inhibitory effect of MEG3 on TERT, indicating that MEG3 is dependent on P53 via H3K27me3. Furthermore, the expression of TERT in human liver cancer stem cells was inhibited. Moreover, MEG3 promotes the binding of P53 and HP1a to telomerase RNA TERC in human hepatoma stem cells, competitively reducing the binding of TERT to TERC, and P53 knockdown can abolish these functions of MEG3. In addition, MEG3 promotes the expression of long non-coding RNA TERRA by altering the activity of DNA methyltransferase DNMT3b, 
increasing the binding of TERT to TERRA. In particular, MEG3 regulates telomere lifespan and is involved in the function of long non-coding RNA HULC, and P53 is directly involved in this process.

First, we found that MEG3 promotes the binding of P300 and RNA pol II to the P53 promoter region. P300 is a histone acetyltransferase that catalyzes the acetylation of histones and promotes gene expression (74). It can be seen that MEG3 may promote the acetylation of histones in the promoter region by increasing the binding of P300 to the P53 promoter, making the chromatin structure loose, allowing more RNA pol II to bind to the P53 promoter region, ultimately increasing transcriptional activity of P53.

Secondly, our study found that MEG3 promotes the interaction of PRC2 complex with histone H3, which in turn promotes methylation of histone H3 lysine 27 in human hepatoma stem cells (H3K27me1/2/3). The PRC2 complex (EZH2, SUZ12, EED, RbAp46/48) is a chromatin-binding complex with histone modification activity, which catalyzes the methylation modification of the 27th lysine of histone $\mathrm{H} 3$. Related to transcriptional repression of target genes (75-77). Studies have shown that abnormalities in the methylation status of PRC2 complexes and histone H3K27 in tumor cells lead to redistribution of H3K27me3, which alters the expression of certain differentiation-related genes, which contributes to the malignant proliferation of tumors (78). For example, high expression of EZH2 in prostate cancer cells increases the level of histone H3K27me3 in the promoter region of the tumor suppressor gene ID4, which in turn promotes methylation modification of the ID4 promoter region by DNMT1 and inhibits transcription of ID4 (79). Therefore, we can speculate that MEG3 promotes the binding of PRC2 complex to histone $\mathrm{H} 3$, which may lead to redistribution of histone H3K27 methylation in the gene, which will change the expression of certain genes, such as reverse transcriptase TERT.

Furthermore, we also found that MEG3 enhances the interaction of the heterochromatin protein HP1a with DNMT3b in human hepatoma stem cells, competitively attenuating the binding of DNMT3b to the long non-coding RNA TERRA promoter, thereby promoting TERRA in human liver cancer stem cells. expression. DNMT3b is a de novo synthetic DNA methyltransferase that catalyzes methylation of $\mathrm{CpG}$ and the fifth carbon atom of cytosine of non-CpG dinucleotides(80). Typically, methylation of DNA in the promoter region of a gene results in silencing of expression of the gene. Studies have shown that DNA methyltransferase is highly expressed in a variety of tumors and is often associated with hypermethylation of tumor suppressor DNA (81). In our study, MEG3 probably reduced the expression of TERRA by inhibiting the binding of DNMT3b to the TERRA promoter and reducing DNA methylation in the TERRA promoter region. Therefore, MEG3 altered the expression of TERRA by altering the epigenetic modification of the TERRA promoter region by affecting DNA methyltransferase activity in human liver cancer stem cells.

It is worth noting that the heterochromatin protein HP1a regulates the function of MEG3 in human hepatoma stem cells. Knockdown of HP1a expression in MEG3 overexpressing human hepatoma stem cells inhibits the binding of MEG3 and P53, indicating that HP1a plays a positive regulatory role in the binding of MEG3 and P53. HP1a is a heterochromatin protein that recognizes the methylation status of histone $\mathrm{H} 3 \mathrm{~K} 9$, and then recruits related enzymes to the corresponding site for methylation modification, 
which mediates gene silencing(82). Numerous studies have shown that HP1 a has dual regulatory effects in tumor cells. For example, up-regulation of HP1a expression is associated with accelerated proliferation of primary tumor cells and poor clinical prognosis, while downregulation of HP1a is beneficial for invasion and metastasis of tumor cells during carcinogenesis (83). Several reports have shown that HP1 a can promote the level of histone H3K9me3 in the tumor suppressor gene promoter region of tumor cells, so HP1a plays a role in promoting tumor growth by participating in the regulation of epigenetic modification of tumor-associated genes (84). In this study, we found that HP1 a promotes the binding of MEG3 and P53, suggesting that HP1 a enhances the tumor suppressor function of MEG3 to a certain extent, and suggests that HP1 a may have rich functions during tumorigenesis and development.

\section{Epigenetic regulation of telomere lifespan of human liver cancer stem cells by MEG3}

Telomeres are special nuclear protein structures at the ends of eukaryotic chromosomes that protect chromosome ends from fusion and degradation, thereby maintaining genome stability (85). In most somatic cells, the telomere length of a chromosome becomes shorter as the number of cell divisions increases (86). When the shortened telomeres are not sufficient to protect the chromosome, it will trigger a DNA damage response and regenerative senescence in the cell, and the cell will stop dividing (85). Therefore, maintenance of telomere length is a prerequisite for cells to undergo long-term division.

First, we found that MEG3 inhibits telomerase activity in human liver cancer stem cells. The main evidences include: (a) MEG3 significantly increased the H3K27me3 modification of the telomerase reverse transcriptase TERT promoter, reduced the binding of RNA pol II to the TERT promoter, and ultimately inhibited the expression of TERT at the transcriptional level. (b) MEG3 competitively reduces the binding of TERT to telomerase RNA TERC via P53 and HP1a. (c) MEG3 promotes the binding of P300 and RNA pol II to the long-chain non-coding RNA TERRA promoter, promotes TERRA expression at the transcriptional level, and ultimately inhibits telomerase activity. Studies have shown that TERRA is a longchain non-coding RNA encoded by telomere DNA and dependent on RNA polymerase II (87). TERT is a catalytic subunit of telomerase, TERC is an RNA template during telomere extension, and TERT and TERC together constitute active telomerase (88). Studies have reported that the 5'-UUAGGG-3' repeat of TERRA can bind to telomerase RNA (TERC) through the principle of base-complementary pairing, which can also interact directly with TERT (89). TERRA competitively inhibits the binding of telomerase to telomeric DNA by both methods, thereby inhibiting telomerase activity. Our study found that MEG3 promotes the expression of TERRA and thus enhances the binding of TERRA to TERT, competitively inhibits the interaction of TERC with TERT, and ultimately inhibits telomerase activity in human hepatoma stem cells, consistent with literature reports. .

Strikingly, our study also found that although MEG3 inhibited the growth of human liver cancer stem cells, the excess of telomerase reverse transcriptase TERT abolished the tumor suppressor function of MEG3. Thus, the tumor suppressor function of MEG3 is closely related to the activity of telomerase. Studies have shown that telomerase is a reverse transcriptase that adds telomere repeats to the ends of chromosomes during cell division to maintain telomere length (90). Numerous studies have shown that 
telomerase activity is increased in a variety of tumor cells, and that increased telomerase activity is associated with increased copy number of telomerase core members TERC and TERT genes (91). Therefore, we believe that MEG3 significantly reduces the activity of telomerase in human hepatocellular carcinoma stem cells by inhibiting the expression of telomerase member TERT or by promoting the competitive inhibition of TERRA expression by binding of TERC to TERT. As the number of divisions increases, it gradually shortens, and eventually the cells stop dividing. Thus, MEG3 shortens telomere lifespan by inhibiting telomerase activity and inhibits the growth of human liver cancer stem cells.

Moreover, our results also indicate that MEG3 regulates the length of telomeres in human liver cancer stem cells. The main basis is as follows: (a) MEG3 increased the binding of CST/AAF to telomere protein POT1, and decreased the interaction between TRF2 and SNM1B, and inhibited the formation of telomere length maintenance complex. (b) MEG3 reduces the stability of telomeres by inhibiting the combination of telomere structure by inhibiting telomere length (including POT1, Exo1, TRF2, SNM1B). (c) MEG3 increased the binding of P53 to long-chain non-coding RNA HULC, thereby inhibiting the formation of telomere length maintenance complex and its ability to bind to telomeres. (d) Although MEG3 inhibited the proliferation of human liver cancer stem cells, the excess of the telomere length maintenance protein TRF2 abolished the tumor suppressor function of MEG3. It can be seen that MEG3 reduces the length of telomeres and shortens the telomere life by a variety of mechanisms with the help of P53.

Importantly, our results suggest that MEG3 controls telomere length and is closely related to HULC. Studies have shown that HULC is a long- non-coding RNA that is highly expressed in a variety of tumors and has been shown to be an oncogene that promotes tumor development through a variety of mechanisms (92). In our previous study, we found that HULC can form a complex with the telomere length maintenance protein TRF2 and bind to the telomere structure, replacing the CST/AAF protein on the telomere and recruiting telomere-associated proteins POT1, Exo1, and SNM1B. The stability of telomeres ultimately promotes the malignant proliferation of liver cancer stem cells (93). Our results show that HULC can promote the formation of telomere length maintenance complex and its binding to telomere structure, while HULC knockdown abolishes these functions. We also found that MEG3 significantly promoted the interaction of P53 with HULC in liver cancer stem cells, thereby competitively reducing the binding ability of POT1, Exo1, TRF2, SNM1B and HULC. At the same time, P53 knockdown can increase the binding of POT1, Ex01, TRF2, SNM1B to telomeric DNA. Therefore, MEG3 relies on the interaction of P53 and HULC to inhibit the binding of telomere length maintenance complex POT1-Exo1TRF2-SNM1B to telomeric DNA, which reduces the stability of telomeres in human liver cancer stem cells and ultimately shortens telomere lifespan.

In summary, our studies revealed that MEG3 is down-regulated in human hepatocarcinoma tissues, and MEG3 significantly inhibits the growth of human liver cancer stem cells in vitro and in vivo. In human liver cancer stem cells, MEG3 promotes the expression of P53, and P53 promotes methylation of histone H3 on the 27th lysine, inhibiting the transcriptional activity of TERT. At the same time, MEG3 inhibits telomerase activity in human liver cancer stem cells by reducing the binding of TERT to TERC. In addition, MEG3 promotes the interaction of P53 with HULC in human hepatoma stem cells, inhibits the binding of 
telomere length maintenance complex POT1-Exo1-TRF2-SNM1B to telomeric DNA, and finally inhibits the length of telomeres in human liver cancer stem cells. Therefore, MEG3 inhibits the activity of telomerase in human liver cancer stem cells and shortens the length of telomeres, thereby inhibiting the occurrence and malignant progression of human liver cancer. These results provide important basic data and theoretical basis for the prevention and treatment of human liver cancer. However, we also need to continue to explore a more detailed mechanism of MEG3 action. For example: (a) Analyze the pattern of dynamic binding of MEG3 and P53 in human liver cancer stem cells and their effects on chromosome remodeling by means of crystal structure analysis and other techniques. (b) Further reveal the precise mechanism of MEG3 regulation of telomerase activity and telomere length in human hepatoma stem cells. (c) Expand the analysis of clinical specimens of liver cancer, and summarize the temporal and spatial regularity of MEG3 expression in tumor patients, and further provide evidence for the application of MEG3 in the clinical diagnosis and treatment of liver cancer. (d) Research and development of MEG3based genetic engineering targeted drugs to provide a more effective means for the treatment of liver cancer.

\section{Conclusions}

MEG3 inhibited the growth in vitro and in vivo of hLCSCs by reducing the activity of telomerase and attenuating telomeric repeat binding factor 2(TRF2). our results demonstrated MEG3 inhibits the occurrence of human liver cancer and these findings provide an important insight into the prevention and treatment of human liver cancer.

\section{Abbreviations}

telomerase reverse transcriptase (TERT)

telomerase RNA (TERC)

TERRA (Telomeric Repeat-Containing RNA)

heterochromatin 1 (HP1)

interstitial Heterochromatin (PCH)

polycomb Repressive Complex 2 (PRC2)

the methylation of the 27th lysine of histone H3 (H3K27me2/3)

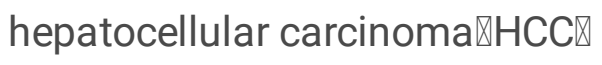

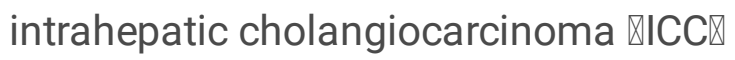

sodium dodecyl sulfate-polyacrylamide gel electrophoresis (SDS-PAGE) 
Chromosome conformation capture -chromatin immunoprecipitation ((3C-ChIP)

proliferating cell nuclear antigen (PCNA)

human hepatoma stem cell lines (hLCSCs)

\section{Declarations}

\section{Ethics approval and consent to participate}

All methods were carried out in "accordance" with the approved guidelines. All experimental protocols "were approved by" a Tongji university institutional committee. Informed consent was obtained from all subjects. The study was reviewed and approved by the China national institutional animal care and use committee.

\section{Consent for publication}

'Not applicable'

\section{Availability of data and material}

'Not applicable'

\section{Competing interests}

"The authors declare that they have no competing interests"

\section{Funding}

This study was supported by grants from National Natural Science Fundation of China(NCSF No.81572773) and National Natural Science Fundation of China (NCSF No.8127229181773158) and by grants from Science and Technology Commission of Shanghai Municipality Basic Research Field Project ه19JC1415200区.

\section{Authors' contributions}

Dongdong Lu conceived the study and participated in the study design, performance, coordination and manuscript writing. Xiaoxue Jiang, Liyan Wang, Sijie Xie, Yingjie Chen, Shuting Song, Yanan Lu performed the research. All authors have read and approved the final manuscript.

\section{Acknowledgements}

This study was supported by grants from National Natural Science Fundation of China (NCSF No.8127229181773158) and by grants from Science and Technology Commission of Shanghai Municipality Basic Research Field Project\19JC1415200囚. 


\section{References}

1.Rosental B, Kowarsky M, Seita J, Corey DM, Ishizuka KJ, Palmeri KJ, Chen SY, Sinha R, Okamoto J, Mantalas G, Manni L, Raveh T, Clarke DN, Tsai JM, Newman AM, Neff NF, Nolan GP, Quake SR, Weissman IL, Voskoboynik A. Complex mammalian-like haematopoietic system found in a colonial chordate.Nature. 2018;564(7736):425-429

2.Messal HA, Alt S, Ferreira RMM, Gribben C, Wang VM, Cotoi CG, Salbreux G, Behrens A. Tissue curvature and apicobasal mechanical tension imbalance instruct cancer morphogenesis. Nature. 2019;566(7742):126-130.

3.Sánchez-Danés A, Larsimont JC, Liagre M, Muñoz-Couselo E, Lapouge G, Brisebarre A, Dubois C, Suppa M, Sukumaran V, Del Marmol V, Tabernero J, Blanpain C. A slow-cycling LGR5 tumour population mediates basal cell carcinoma relapse after therapy.Nature. 2018;562(7727):434-438

4.Mrna Choe J, Lin S, Zhang W, Liu Q, Wang L, Ramirez-Moya J, Du P, Kim W, Tang S, Sliz P, Santisteban P, George RE, Richards WG, Wong KK, Locker N, Slack FJ, Gregory RI. mRNA circularization by METTL3elF3h enhances translation and promotes oncogenesis. Nature. 2018;561(7724):556-560

5.Fu T, Coulter S, Yoshihara E, Oh TG, Fang S, Cayabyab F, Zhu Q, Zhang T, Leblanc M, Liu S, He M, Waizenegger W, Gasser E, Schnabl B, Atkins AR, Yu RT, Knight R, Liddle C, Downes M, Evans RM. FXR Regulates Intestinal Cancer Stem Cell Proliferation. Cell. 2019;176(5):1098-1112.

6.Gronke K, Hernández PP, Zimmermann J, Klose CSN, Kofoed-Branzk M, Guendel F, Witkowski M, Tizian C, Amann L, Schumacher F, Glatt H, Triantafyllopoulou A, Diefenbach A. Interleukin-22 protects intestinal stem cells against genotoxic stress. Nature. 2019;566(7743):249-253

7.Bahr C, von Paleske L, Uslu VV, Remeseiro S, Takayama N, Ng SW, Murison A, Langenfeld K, Petretich M, Scognamiglio R, Zeisberger P, Benk AS, Amit I, Zandstra PW, Lupien M, Dick JE, Trumpp A, Spitz F. A Myc enhancer cluster regulates normal and leukaemic haematopoietic stem cell hierarchies. Nature. 2018;553(7689):515-520.

8. Xu XL, Xing BC, Han HB, et al. The properties of tumor-initiating cells from a hepatocellular carcinoma patient's primary and recurrent tumor. Carcinogenesis, 2010, 31(2): 167-174.

9.Blackburn EH. Structure and function of telomeres. Nature, 1991, 350(6319): 569-573.

10.Palm W, de Lange T. How shelterin protects mammalian telomeres. Annual review of genetics, 2008, 42: 301-334.

11.Galati A, Micheli E, Alicata C, et al. TRF1 and TRF2 binding to telomeres is modulated by nucleosomal organization. Nucleic acids research, 2015, 43(12): 5824-5837. 
12.Stone MD. Detailed view of human telomerase enzyme invites rethink of its structure. Nature. 2018;557(7704):174-175

13.Blackburn EH EE, Lin J. Human telomere biology: A contributory and interactive factor in aging, disease risks, and protection. Science, 2015, 350(6265): 1193-1198.

14.Shay JW, Bacchetti S. A survey of telomerase activity in human cancer. European journal of cancer, 1997, 33(5): 787-791.

15.Zaret KS. The telomerase enzyme and liver renewal. Nature. 2018;556(7700):181-182

16.Wang S, Pike AM, Lee SS, et al. BRD4 inhibitors block telomere elongation. Nucleic acids research, 2017, 45(14): 8403-8410.

17.Benetti R, Garcia-Cao M, Blasco MA. Telomere length regulates the epigenetic status of mammalian telomeres and subtelomeres. Nature genetics, 2007, 39(2): 243-250.

18.Azzalin CM, Reichenbach P, Khoriauli L, et al. Telomeric repeat containing RNA and RNA surveillance factors at mammalian chromosome ends. Science, 2007, 318(5851): 798-801.

19.Cusanelli E, Chartrand P. Telomeric repeat-containing RNA TERRA: a noncoding RNA connecting telomere biology to genome integrity. Frontiers in genetics, 2015, 6: 143.

20.Deng Z, Norseen J, Wiedmer A, et al. TERRA RNA binding to TRF2 facilitates heterochromatin formation and ORC recruitment at telomeres. Molecular cell, 2009, 35(4): 403-413.

21.Redon S, Reichenbach P, Lingner J. The non-coding RNA TERRA is a natural ligand and direct inhibitor of human telomerase. Nucleic acids research, 2010, 38(17): 5797-5806.

22.Redon S, Zemp I, Lingner J. A three-state model for the regulation of telomerase by TERRA and hnRNPA1. Nucleic acids research, 2013, 41(19): 9117-9128.

23.Zhang JM, Yadav T, Ouyang J, Lan L, Zou L. Alternative Lengthening of Telomeres through Two Distinct Break-Induced Replication Pathways. Cell Rep. 2019;26(4):955-968

24.Dilley RL, Verma P, Cho NW, Winters HD, Wondisford AR, Greenberg RA. Break-induced telomere synthesis underlie alternative telomere maintenance. Nature. 2016;539(7627):54-58

25.Noordermeer SM, Adam S, Setiaputra D, Barazas M, Pettitt SJ, Ling AK, Olivieri M, Álvarez-Quilón A, Moatti N, Zimmermann M, Annunziato S, Krastev DB, Song F, Brandsma I, Frankum J, Brough R, Sherker A, Landry S, Szilard RK, Munro MM, McEwan A, Goullet de Rugy T, Lin ZY, Hart T, Moffat J, Gingras AC, Martin A, van Attikum H, Jonkers J, Lord CJ, Rottenberg S, Durocher D. The shieldin complex mediates 53BP1-dependent DNA repair.Nature. 2018;560(7716):117-121

26.Zlotorynski E. Telomerecrisis activates autophagic death. Nat Rev MolCell Biol. 2019;20(3):133 
27.Cubiles MD, Barroso S, Vaquero-Sedas MI, Enguix A, Aguilera A, Vega-Palas MA. Epigenetic features of human telomeres. Nucleic Acids Res. 2018;46(5):2347-2355

28.Schmidt JC, Zaug AJ, Cech TR. Live Cell Imaging Reveals the Dynamics of Telomerase Recruitment to Telomeres. Cell 2016;166(5):1188-1197

29.Zhou Y, Cheunsuchon P, Nakayama Y, et al. Activation of paternally expressed genes and perinatal death caused by deletion of the Gtl2 gene. Development, 2010, 137(16): 2643-2652.

30.Stadtfeld M, Apostolou E, Akutsu H, Fukuda A, Follett P, Natesan S, Kono T, Shioda T, Hochedlinger K. Aberrant silencing of imprinted genes on chromosome 12qF1 in mouse induced pluripotent stem cells. Nature 2010;465(7295):175-81

31.Yu F, Geng W, Dong P, Huang Z, Zheng J.LncRNA-MEG3 inhibits activation of hepatic stellate cells through SMO protein and miR-212. Cell Death Dis. 2018;9(10):1014

32. Huang P, Huang FZ, Liu HZ, Zhang TY, Yang MS, Sun CZ. LncRNA MEG3 functions as a ceRNA in regulating hepatic lipogenesis by competitively binding to miR-21 with LRP6.Metabolism. 2019 Jan 31. pii: S0026-0495(19)30033-2.

33.Zhang X, Rice K, Wang Y, et al. Maternally expressed gene 3 (MEG3) noncoding ribonucleic acid: isoform structure, expression, and functions. Endocrinology, 2010, 151(3): 939-947.

34.Zhou Y, Zhang X, Klibanski A. MEG3 noncoding RNA: a tumor suppressor. Journal of molecular endocrinology, 2012, 48(3): R45-53.

Yan J, Guo X, Xia J, et al. MiR-148a regulates MEG3 in gastric cancer by targeting DNA methyltransferase 1. Medical oncology, 2014, 31(3): 879.

36.Liu LX, Deng W, Zhou XT, et al. The mechanism of adenosine-mediated activation of IncRNA MEG3 and its antitumor effects in human hepatoma cells. International journal of oncology, 2016, 48(1): 421429 .

37.Zhou Y, Zhong Y, Wang Y, et al. Activation of p53 by MEG3 non-coding RNA. The Journal of biological chemistry, 2007, 282(34): 24731-24742.

38.Wu M, Huang Y, Chen T, Wang W, Yang S, Ye Z, Xi X. LncRNA MEG3 inhibits the progression of prostate cancer by modulating miR-9-5p/QKI-5 axis.J Cell Mol Med. 2019;23(1):29-38

39.Zhang Y, Wu J, Jing H, Huang G, Sun Z, Xu S. Long noncoding RNA MEG3 inhibits breast cancer growth via upregulating endoplasmic reticulum stress and activating NF-KB and p53.J Cell Biochem. 2019;120(4):6789-6797 
40.Jiang M, Wang YR, Xu N, Zhou L, An Q. Long noncoding RNA MEG3 play an important role in osteosarcoma development through sponging microRNAs.J Cell Biochem. 2019;120(4):5151-5159.

41.Lavin MF, Gueven N. The complexity of p53 stabilization and activation. Cell death and differentiation, 2006, 13(6): 941-950.

42. Harris SL, Levine AJ. The p53 pathway: positive and negative feedback loops. Oncogene, 2005, 24(17): 2899-2908.

43.Vousden KH, Prives C. Blinded by the Light: The Growing Complexity of p53. Cell, 2009, 137(3): 413431.

44.Ghezraoui H, Oliveira C, Becker JR, Bilham K, Moralli D, Anzilotti C, Fischer R, Deobagkar-Lele M, Sanchiz-Calvo M, Fueyo-Marcos E, Bonham S, Kessler BM, Rottenberg S, Cornall RJ, Green CM, Chapman JR. 53BP1 cooperation with the REV7-shieldin complex underpins DNA structure-specific NHEJ.Nature. 2018;560(7716):122-127

45.Maya-Mendoza A, Moudry P, Merchut-Maya JM, Lee M, Strauss R, Bartek J. High speed of fork progression induces DNA replication stress and genomic instability. Nature. 2018;559(7713):279-284

46.Canzio D, Larson A, Narlikar GJ. Mechanisms of functional promiscuity by HP1 proteins. Trends in cell biology, 2014, 24(6): 377-386.

47 Maison C, Almouzni G. HP1 and the dynamics of heterochromatin maintenance. Nature reviews Molecular cell biology, 2004, 5(4): 296-304.

48.Eissenberg JC, Elgin SC. HP1a: a structural chromosomal protein regulating transcription. Trends in genetics : TIG, 2014, 30(3): 103-110.

49.Bosch-Presegue L, Raurell-Vila H, Thackray JK, et al. Mammalian HP1 Isoforms Have Specific Roles in Heterochromatin Structure and Organization. Cell reports, 2017, 21(8): 2048-2057.

50.Jensen KN, Lorincz MC. HP1 proteins safeguard embryonic stem cells. Nature. 2018;557(7707):640641

51.Ostapcuk V, Mohn F, Carl SH, Basters A, Hess D, lesmantavicius V, Lampersberger L, Flemr M, Pandey A, Thomä NH, Betschinger J, Bühler M. Activity-dependent neuroprotective protein recruits HP1 and CHD4 to control lineage-specifying genes. Nature. 2018;557(7707):739-743

52.Vizan P, Beringer M, Ballare $\mathrm{C}$, et al. Role of PRC2-associated factors in stem cells and disease. The FEBS journal, 2015, 282(9): 1723-1735.

53.Margueron R, Reinberg D. The Polycomb complex PRC2 and its mark in life. Nature, 2011, 469(7330): 343-349. 
54 Liu YL, Gao X, Jiang Y, et al. Expression and clinicopathological significance of EED, SUZ12 and EZH2 mRNA in colorectal cancer. Journal of cancer research and clinical oncology, 2015, 141(4): 661-669.

55.Manji SS, Sorensen BS, Klockars TI. Molecular characterization and expression of maternally expressed gene 3 (Meg3/Gtl2) RNA in the mouse inner ear. Journal of neuroscience research, 2006, 83(2): 181-190.

56.Zhou Y, Cheunsuchon P, Nakayama Y. Activation of paternally expressed genes and perinatal death caused by deletion of the Gtl2 gene. Development, 2010, 137(16): 2643-2652.

57.He Y, Wu YT, Huang C. Inhibitory effects of long noncoding RNA MEG3 on hepatic stellate cells activation and liver fibrogenesis. Biochimica et biophysica acta, 2014, 1842(11): 2204-2215.

58.Chak WP, Lung RW, Tong JH. Downregulation of long non-coding RNA MEG3 in nasopharyngeal carcinoma. Molecular carcinogenesis, 2017, 56(3): 1041-1054.

59.Ying $L$, Huang $Y$, Chen $\mathrm{H}$, et al. Downregulated MEG3 activates autophagy and increases cell proliferation in bladder cancer. Molecular bioSystems, 2013, 9(3): 407-411.

60.Sun M, Xia R, Jin F, et al. Downregulated long noncoding RNA MEG3 is associated with poor prognosis and promotes cell proliferation in gastric cancer. Tumour biology: the journal of the International Society for Oncodevelopmental Biology and Medicine, 2014, 35(2): 1065-1073.

61.Lu KH LW, Liu XH, Sun M, et al. Long non-coding RNA MEG3 inhibits NSCLC cells proliferation and induces apoptosis by affecting p53 expression. BMC Cancer, 2013, 13: 461.

62.Li J, Bian EB, He XJ, et al. Epigenetic repression of long non-coding RNA MEG3 mediated by DNMT1 represses the p53 pathway in gliomas. International journal of oncology, 2016, 48(2): 723-733.

63.] Qin R, Chen Z, Ding Y, et al. Long non-coding RNA MEG3 inhibits the proliferation of cervical carcinoma cells through the induction of cell cycle arrest and apoptosis. Neoplasma, 2013, 60(5): 486492.

64.Guo Q, Qian Z, Yan D, et al. LncRNA-MEG3 inhibits cell proliferation of endometrial carcinoma by repressing Notch signaling. Biomedicine \& pharmacotherapy, 2016, 82: 589-594.

65.Xu XL, Xing BC, Han HB, et al. The properties of tumor-initiating cells from a hepatocellular carcinoma patient's primary and recurrent tumor. Carcinogenesis, 2010, 31(2): 167-174.

67.Li J, Chen JN, Zeng TT, et al. CD133+ liver cancer stem cells resist interferon-gamma-induced autophagy. BMC cancer, 2016, 16: 15.

67.Pflaum J, Schlosser S, Muller M. p53 Family and Cellular Stress Responses in Cancer. Frontiers in oncology, 2014, 4: 285. 
68.Pflaum J, Schlosser S, Muller M. p53 Family and Cellular Stress Responses in Cancer. Frontiers in oncology, 2014, 4: 285.

69 Lavin MF, Gueven N. The complexity of p53 stabilization and activation. Cell death and differentiation, 2006, 13(6): 941-950.

70.Basu S, Murphy ME. Genetic Modifiers of the p53 Pathway. Cold Spring Harbor perspectives in medicine, 2016, 6(4): a026302.

71.Liou AT, Chen MF, Yang CW. Curcumin Induces p53-Null Hepatoma Cell Line Hep3B Apoptosis through the AKT-PTEN-FOXO4 Pathway. Evidence-based complementary and alternative medicine : eCAM, 2017, 2017: 4063865.

72.Soussi T, Lozano G. p53 mutation heterogeneity in cancer. Biochemical and biophysical research communications, 2005, 331(3): 834-842

73.Deshmukh A, Binju M, Arfuso F, et al. Role of epigenetic modulation in cancer stem cell fate. The international journal of biochemistry \& cell biology, 2017, 90: 9-16.

74.Chan WM, Mak MC, Fung TK, et al. Ubiquitination of p53 at multiple sites in the DNA-binding domain. Molecular cancer research: MCR, 2006, 4(1): 15-25

75.Vizan P, Beringer M, Ballare C, et al. Role of PRC2-associated factors in stem cells and disease. The FEBS journal, 2015, 282(9): 1723-1735.

76.Margueron R, Reinberg D. The Polycomb complex PRC2 and its mark in life. Nature, 2011, 469(7330): 343-349.

77.Margueron R, Li G, Sarma K, et al. Ezh1 and Ezh2 maintain repressive chromatin through different mechanisms. Molecular cell, 2008, 32(4): 503-518.

78.Conway E, Healy E, Bracken AP. PRC2 mediated H3K27 methylations in cellular identity and cancer. Current opinion in cell biology, 2015, 37: 42-48.

79.Chinaranagari S, Sharma P, Chaudhary J. EZH2 dependent H3K27me3 is involved in epigenetic silencing of ID4 in prostate cancer. Oncotarget, 2014, 5(16): 7172-7182.

80.Uysal F, Akkoyunlu G, Ozturk S. Dynamic expression of DNA methyltransferases (DNMTs) in oocytes and early embryos. Biochimie, 2015, 116: 103-113.

81.Esteller M. Epigenetics in cancer. The New England journal of medicine, 2008, 358(11): 1148-1159.

82.Eissenberg JC, Elgin SC. HP1a: a structural chromosomal protein regulating transcription. Trends in genetics: TIG, 2014, 30(3): 103-110. 
83.Kirschmann DA, Lininger RA, Gardner LM, et al. Down-regulation of HP1Hsalpha expression is associated with the metastatic phenotype in breast cancer. Cancer research, 2000, 60(13): 3359-3363.

84.Cheng W, Tian L, Wang B, et al. Downregulation of HP1alpha suppresses proliferation of cholangiocarcinoma by restoring SFRP1 expression. Oncotarget, 2016, 7(30): 48107-48119.

85.Wang $\mathrm{L}$, Xiao H, Zhang $\mathrm{X}$, et al. The role of telomeres and telomerase in hematologic malignancies and hematopoietic stem cell transplantation. Journal of hematology \& oncology, 2014, 7: 61.

86. Harley $\mathrm{CB}$, Futcher $\mathrm{AB}$, Greider $\mathrm{CW}$. Telomeres shorten during ageing of human fibroblasts. Nature, 1990, 345(6274): 458-460.

87.Azzalin CM, Reichenbach P, Khoriauli L, et al. Telomeric repeat containing RNA and RNA surveillance factors at mammalian chromosome ends. Science, 2007, 318(5851): 798-801.

88.Blackburn EH EE, Lin J. Human telomere biology: A contributory and interactive factor in aging, disease risks, and protection. Science, 2015, 350(6265): 1193-1198.

89.Redon S, Reichenbach P, Lingner J. The non-coding RNA TERRA is a natural ligand and direct inhibitor of human telomerase. Nucleic acids research, 2010, 38(17): 5797-5806.

90.Greider CW, Blackburn EH. A telomeric sequence in the RNA of Tetrahymena telomerase required for telomere repeat synthesis. Nature, 1989, 337(6205): 331-337.

91.Shay JW, Bacchetti S. A survey of telomerase activity in human cancer. European journal of cancer, 1997, 33(5): 787-791.

92.Yu X, Zheng H, Chan MT, et al. HULC: an oncogenic long non-coding RNA in human cancer. Journal of cellular and molecular medicine, 2017, 21(2): 410-417.

93.Wu M, Lin Z, Li X, et al. HULC cooperates with MALAT1 to aggravate liver cancer stem cells growth through telomere repeat-binding factor 2. Scientific reports, 2016, 6: 36045.

\section{Figures}


Figure1

A
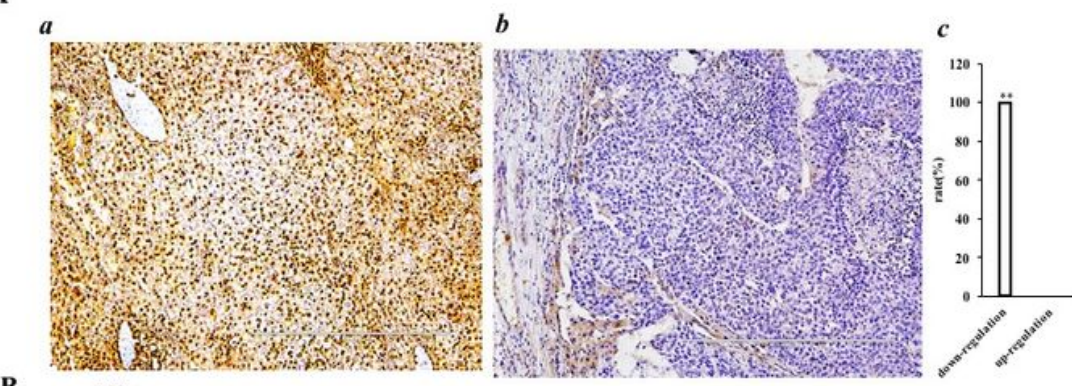

C

D

B
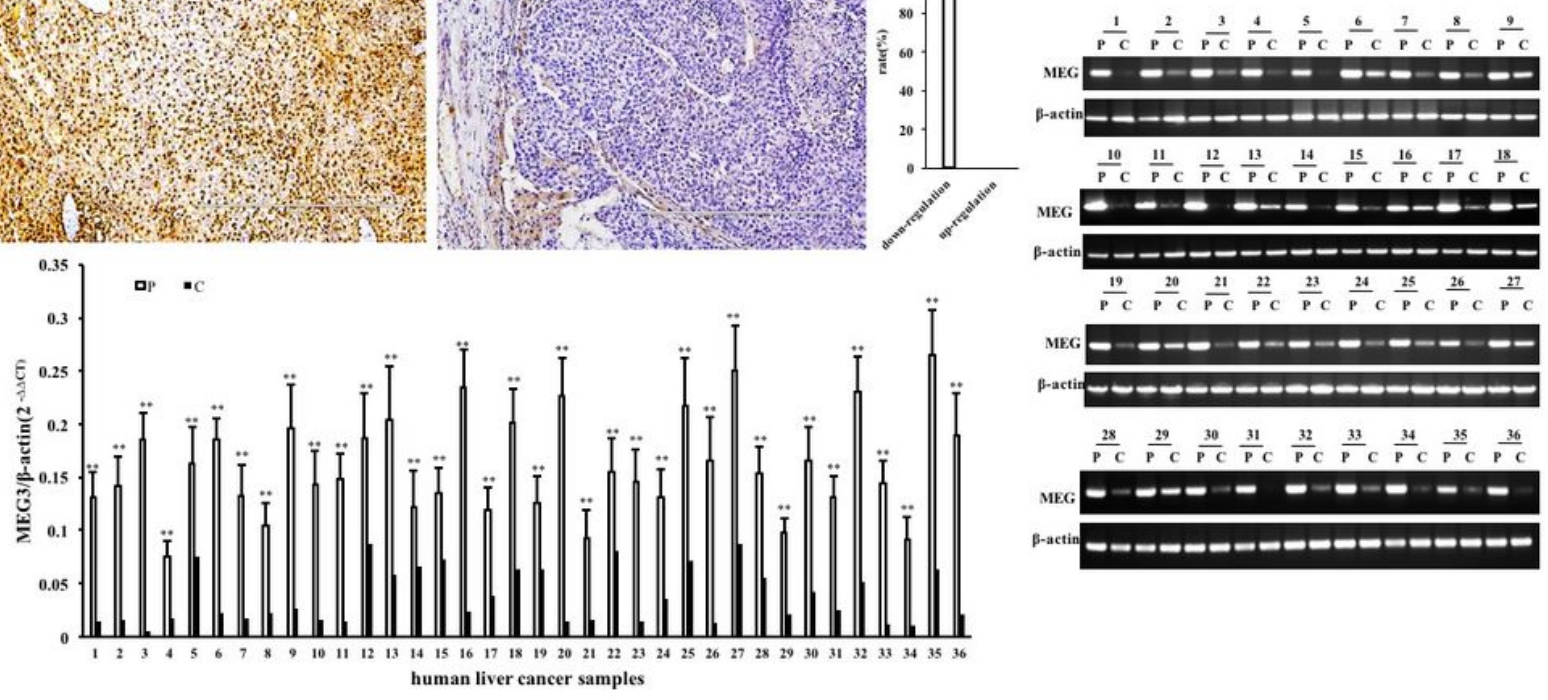
$\frac{19}{\mathrm{PC}} \frac{20}{\mathrm{PC}} \frac{21}{\mathrm{PCC}} \frac{22}{\mathrm{PC}} \frac{23}{\mathrm{PC}} \frac{24}{\mathrm{P}} \mathrm{C} \frac{25}{\mathrm{P}} \mathrm{C} \frac{26}{\mathrm{P}} \mathrm{C} \frac{27}{\mathrm{P} C}$

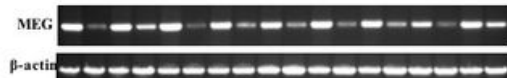
$\frac{28}{\mathrm{PCC}} \frac{29}{\mathrm{PC}} \frac{30}{\mathrm{PCC}} \frac{31}{\mathrm{PCC}} \frac{32}{\mathrm{PC}} \frac{33}{\mathrm{P} C} \frac{34}{\mathrm{PC}} \frac{35}{\mathrm{PC}} \frac{36}{\mathrm{PC}}$
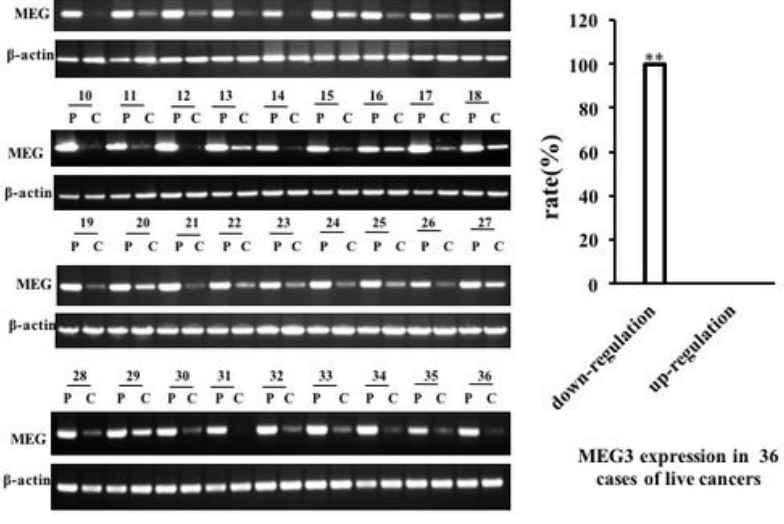

Figure 1

Figures 1-8: Each value was presented as mean \pm standard error of the mean (SEM). Bar \pm SEM. **, $P<0.01 ; *, P<0.05$. For all Western blotting, we repeated the experiments for three times. We measured gray value of the bands for quantification. Each value was presented as mean \pm standard error of the mean (SEM) (Student's t-test) Figure 1 Expression analysis of MEG3 in human liver cancer A. a\&b. Representative results of in situ hybridization analysis of MEG3 expression in human liver cancer and its paracancerous tissue (biotinylated MEG3 probe, DAB staining, 100-folds magnification). a: liver cancer tissue, and b: the adjacent noncancerous liver tissue. c. Down-regulated expression rate of MEG3 in 62 liver cancer patients. B. The results of qRT-PCR analysis of MEG3 expression in fresh human liver cancer and its adjacent tissues. The liver cancer tissue was indicated by $C$, and the adjacent noncancerous liver tissue was indicated by $P$, and the internal reference gene was $\beta$-actin. Each group of values was expressed as mean \pm standard deviation (mean $\pm S E M, n=36$ ). C. RT-PCR analysis of MEG3 expression in human liver cancer and its adjacent noncancerous liver tissues. The liver cancer tissue was indicated by $C$, and the paracancerous tissue was indicated by $P$, and the expression of $\beta$-actin was used as an internal reference. b. Down-regulated expression rate of MEG3 in 36 human hepatocarcinoma tissues and adjacent tissues. Each group of values is expressed as mean \pm standard deviation (mean $\pm S E M, n=36$ ). 
Figure2

A

B

C

D

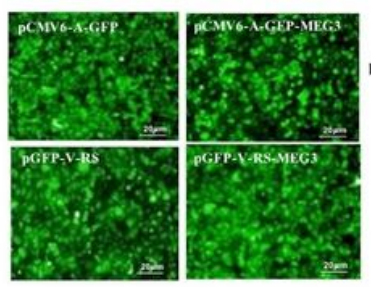

D

E
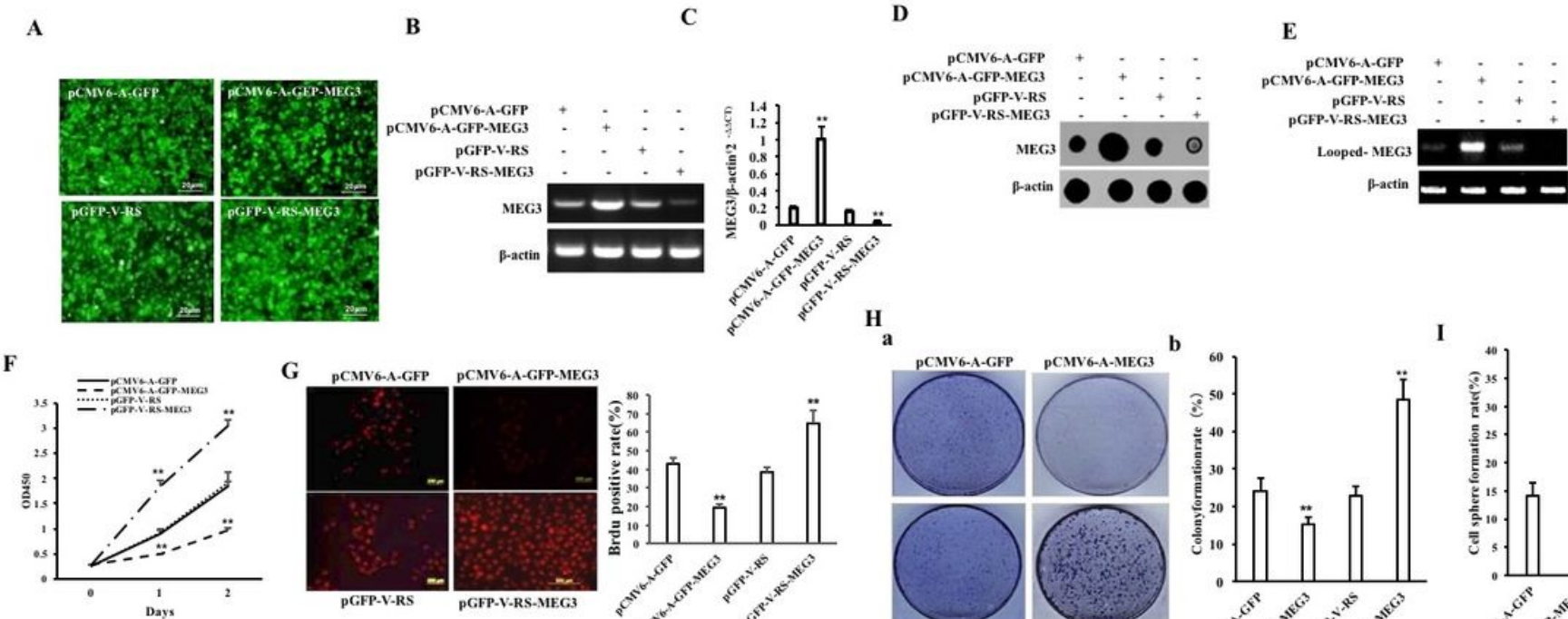

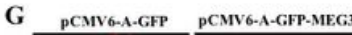
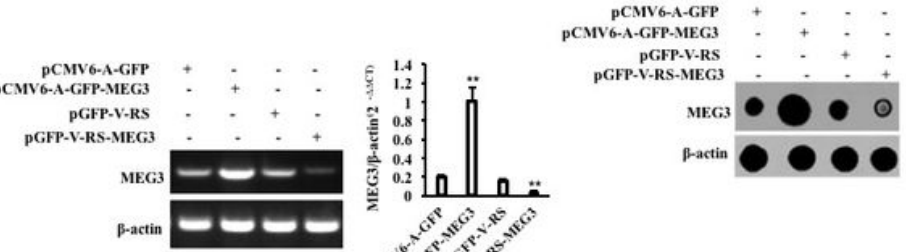

PCMV6-A-GFP-MEC

PGFP-V.RS

(n)

स
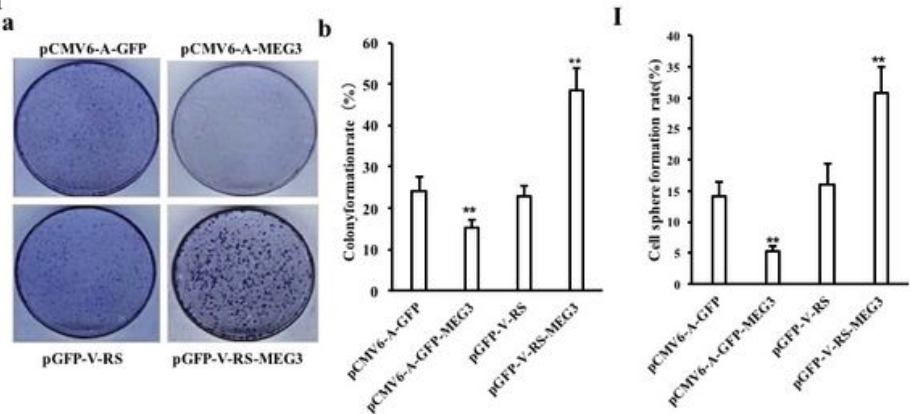

M

L
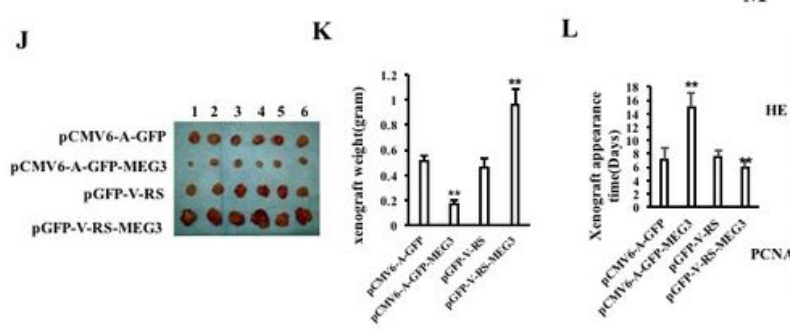

PCMVG-A-GFP

pCMV6-A-MEG3

PGFP.V-RS PGFP-V-RS-MEG3

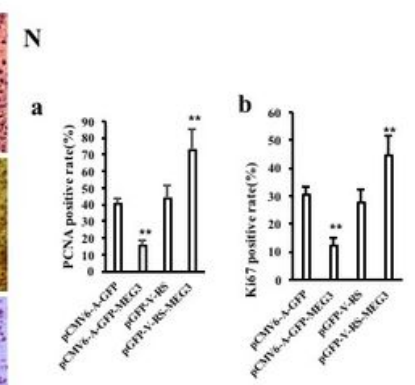

\section{Figure 2}

MEG3 affects tumorigenesis of human liver cancer stem cells in vitro and vivo A. Four stable cell lines were picked up under fluorescence microscopy (including pCMV6-A-GFP-hLCSCs, pCMV6-A-GFP-MEG3hLCSCs, pGFP-V-RS-hLCSCs, and pGFP-V-RS-MEG3). Positive hLCSCs cells expressing green fluorescent protein were expanded and cultured, and images were taken using a fluorescence microscope. $\mathrm{B}$. The expression of MEG3 was detected by RT-PCR in four stable cell lines, and $\beta$-actin was used as an internal reference gene. $C$. The expression of MEG3 was detected by qRT-PCR in four stable cell lines, and $\beta$-actin was used as an internal reference gene. D. Dot blotting was used to detect the expression of MEG3 in four stable cell lines, and $\beta$-actin was used as an internal reference gene. E. The expression of circular MEG3 was detected by back-to-back RT-PCR in four stable cell lines, and $\beta$-actin was used as an internal reference gene.F. The cell proliferation ability of four stably transfected cell lines in 96-well culture plates was determined by CCK8 method. G. a. BrdU stained photo. b. Determine the percentage of S phase cells in four stable transfected cell lines. C. a. Photograph of cell colonies. b. Cell colony formation rate. H. Determination of sphere formation ability of four stable cell lines using microsphere culture plates. I.Human tumor stem cells stably transfected with pCMV6-A-GFP, pCMV6-A-GFP-MEG3, pGFP-V-RS and pGFP-V-RS-MEG3 plasmids were inoculated into Balb/C nude mice. The photographs of dissected transplanted tumors for one month. J. Comparison of weight $(\mathrm{g})$ of transplanted tumors in nude mice. K. Comparison of time (days) of transplanted tumors in nude mice. L. transplanted tumor tissue sections (4 
$\mu \mathrm{m})$ from nude mouse were subjected to hematoxylin-eosin staining, immunohistochemical staining of anti-PCNA and anti-Ki67. M. a. Comparison of PCNA positive rates in transplanted tumors in nude mice. b. Comparison of Ki67 positive rates in transplanted tumors in nude mice.

Figure 3
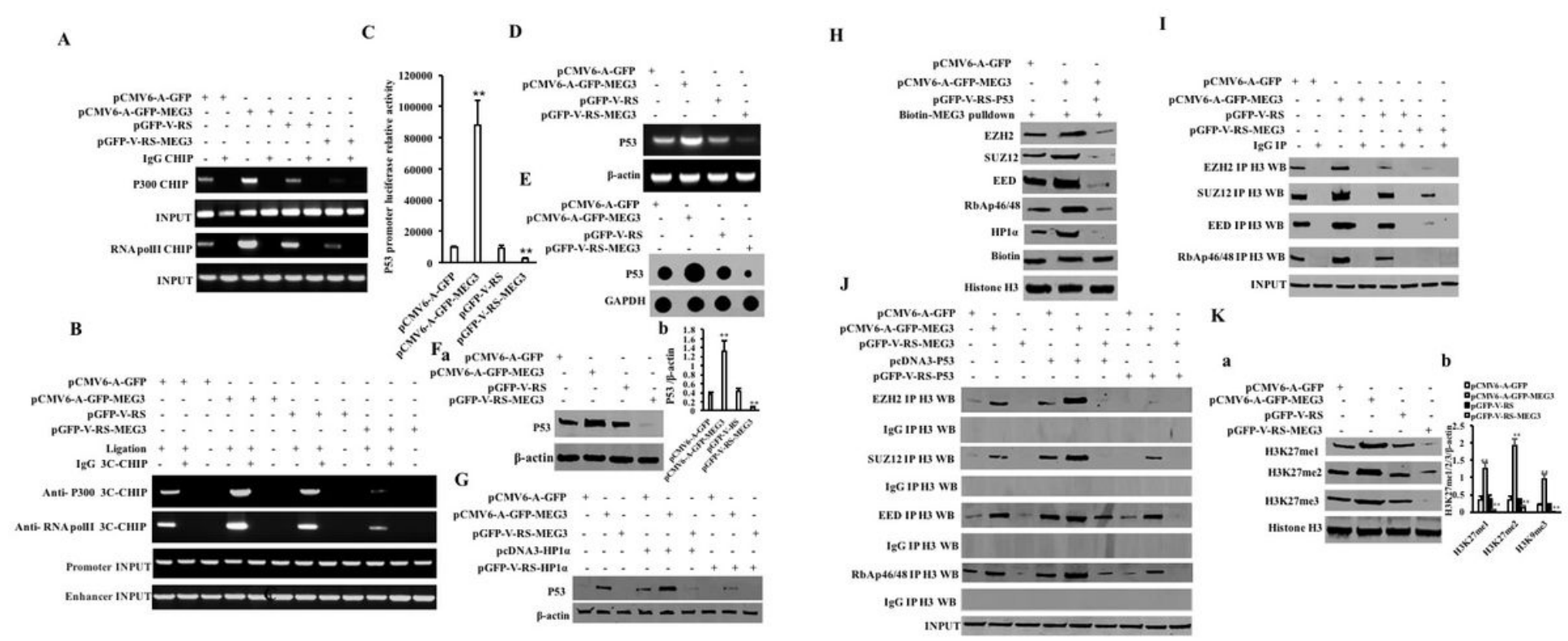

Figure 3

MEG3 affects the P53 expression and MEG3-dependent P53 promotes methylation $\mathrm{n}$ of histone $\mathrm{H} 3$ at lysine 27 in human liver cancer stem cells in liver cancer stem cells. A. Chromatin immunoprecipitation (CHIP) analysis of the P53 promoter in liver cancer stem cells. Human liver cancer stem cell lines (hLCSCs) were used to extract cross-linked DNA, and chromosome co-immunoprecipitation (CHIP) was performed using anti-P300 and anti-RNA polll. The polymers isolated and purified from the CHIP precipitates were used as a template, and a polymerase chain reaction (PCR) amplification was carried out using a primer designed according to the P53 promoter. IgG CHIP was used as a negative control; the DNA retained before chromatin immunoprecipitation was used as a template, and the product amplified by the primer designed by the P53 promoter was used as an internal reference (INPUT).B. Analysis of the binding ability of P300, RNApollI to P53 promoter-enhancer loop in four stable human live cancer stem cell lines (including pCMV6-A-GFP-hLCSCs, pCMV6-A-GFP-MEG3-hLCSCs,pGFP-V-RS-hLCSCs, pGFP-V-RSMEG4-hLCSCs) by chromosomal conformation capture (3C)-chromatin immunoprecipitation (CHIP).The hLCSCs were cross-linked with formaldehyde and then captured by chromosome configuration (3C)chromatin immunoprecipitation (CHIP) using anti-P300, anti-RNAPolll. Polymerase chain reaction (PCR) amplification was carried out using a pair of mixed primers designed according to the P53 promoter and enhancer using the isolated and purified DNA in the CHIP-3C precipitate as a template. IgG CHIP-3C was used as a negative control; the DNA retained by chromatin immunoprecipitation was used as a template, and the products amplified by primers designed by P53 promoter and enhancer were used as internal reference (INPUT).C. The pEZX-MT-P53 promoter-Luc dual luciferase activity was tested. .D. The reverse transcription polymerase chain reaction (RT-PCR) to detect the transcriptional capacity of P53 in these 
four stable cell lines. $\beta$-actin was used as an internal reference. gene.E. The transcriptional ability of P53 in these four stable cell lines was detected by Dot-blot, and GAPDH was used as an internal reference gene.F. a. The translational ability of P53 in these four stable cell lines was detected by Western blotting, and $\beta$-actin was used as an internal reference gene. b. Semi-quantitative analysis of grayscale scanning of positive bands. G. the total protein was extracted and subjected to anti-P53 Western blotting analysis. $\beta$-actin was used as an internal reference gene. H.The RNA pulldown analysis was performed using MEG3 biotinylated probes and anti- EZH2, anti-SUZ12, anti-EDD, and anti-RbAp46/48 in hLCSCs transfected with pCMV6-A-GFP, pCMV6-A-GFP-MEG3, pGFP-V-RS-MEG3, pCMV6-A-GFP-MEG3+ pGFP-VRS-P53. Hisone H3 is used as INPUT and Biotin is used as an internal reference.l. Co-immunoprecipitated with anti-EZH2, anti-SUZ12, anti-EED, anti-RbAp46/48, and anti-Histone H3 was performed. IgG coimmunoprecipitation was used as a negative control, and anti-Histone $\mathrm{H} 3$ in the sample before coprecipitation was subjected to Western blotting as INPUT. J. Co-immunoprecipitation with anti-EZH2, anti-SUZ12, anti-EED, anti-RbAp46/48, and anti-HistoneH3 was performed. IgG co-immunoprecipitation was used as a negative control, and anti-Histone $\mathrm{H} 3$ in the sample before coprecipitation was subjected to Western blotting as INPUT. K.a Total cellular proteins were extracted and the H3K27me1,H3K27me2 and H3K27me3 were detected by Western blotting, and Histone H3 was used as an internal reference gene. b. Semi-quantitative analysis of grayscale scanning of positive bands.

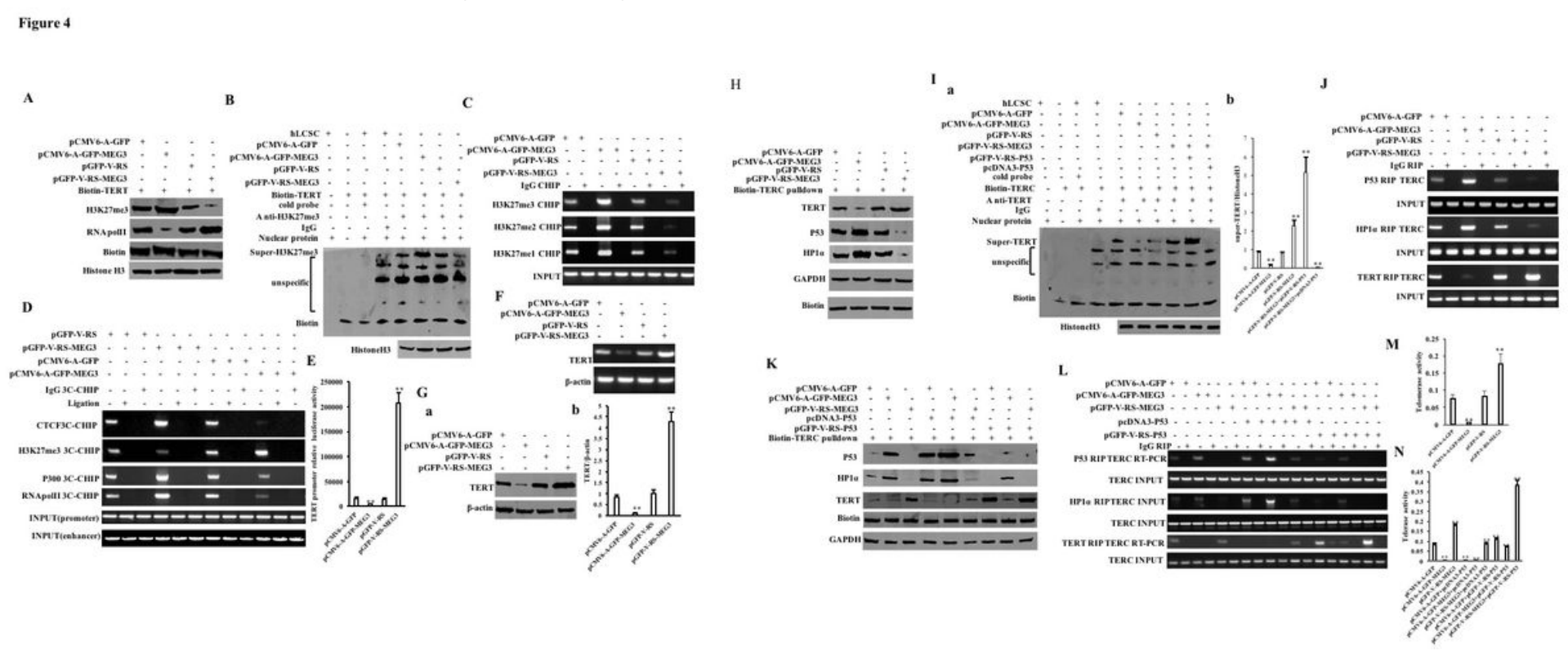

\section{Figure 4}

MEG3 inhibits the expression of telomerase reverse transcriptase gene in human liver cancer stem cells. A. DNA pull down was detected by biotin-labeled TERT promoter probe (Biotin-TERT promoter DNA), and Western blotting analysis was performed with anti-RNA po I II and anti-H3K27me3, respectively. Western blotting of anti-Biotin was used as an internal reference (INPUT) for the amount of probe added, and Western blotting of anti-Histone $\mathrm{H} 3$ was performed as an internal reference.B. Super-DNA-protein complex gel migration assay (Super-EMSA) with Biotin labeled TERT promoter cis-element probe and antiHK27me3, anti-Biotin, IgG super-EMSA as a negative control, nucleoprotein-free EMSA and excessive cold probe was used as a system reference.C. The chromatin immunoprecipitation (CHIP) of anti-H3K27me1, 
anti-H3K27me2 and anti-H3K27me3. The purified DNA is used as a template, and polymerase chain reaction (PCR) amplification is carried out using primers designed to be able to bind to the promoter region of the above-mentioned protein. IgG chromatin immunoprecipitation was used as a negative control; the promoter of TERT was amplified by using the DNA retained before chromatin immunoprecipitation as a template (using the same primer as above) as an internal reference (INPUT).D. The cells were cross-linked with formaldehyde and then captured by chromosome configuration (3C)chromatin immunoprecipitation (CHIP) using anti-CTCF, anti-H3K27me3, anti-RNAPolll. Polymerase chain reaction (PCR) amplification was carried out using a pair of mixed primers designed according to the TERT promoter and enhancer. IgG CHIP-3C was used as a negative control.E. The pEZX-MT-TERT promoter-Luc dual luciferase activity was then tested.F. The transcriptional capacity of TERT was detected in these four stable cell lines by reverse transcription polymerase chain reaction (RT-PCR). $\beta$ actin was used as an internal reference. gene.G.a The total cellular proteins were extracted and the expression of TERT in these four stable cell lines was detected by Western blotting. $\beta$-actin was used as an internal reference gene. b. Semi-quantitative analysis of grayscale scanning of positive bands. H. RNA pull down was detected by biotin-labeled TERC RNA probe (Biotin-TERT), and Western blotting was performed with anti-TERT, anti-HP1 $\alpha$ and anti-P53, respectively. Western blotting of anti-Biotin was used as an INPUT and Western blotting of anti-HistoneH3 was performed as an internal reference of the reaction nuclear protein by using the protein extract before RNA pull down.I. a.The Biotin-labeled TERC probe (Biotin-TERC) and anti-TERT, anti-Biotin were used for Super-RNA-protein complex gel migration assay (Super-EMSA). IgG Super-EMSA was used as a negative control, nucleoprotein-free EMSA and EMSA with excess cold probe added as a system reference, Biotin-TERC hybridization band and the amount of nucleoprotein added as INPUT. b. Quantitative analysis of gray scales of positive bands.J. The RNA immunoprecipitation (RIP) using anti-TERT, anti-P53, anti-HP1a .The RNA purified from the coimmunoprecipitate was used as a template, and the TERC was amplified by RT-PCR using primers designed by the TERC sequence. IgG RNA co-immunoprecipitation was used as a negative control, and TERC was separately amplified as an internal reference (INPUT) using RNA retained before RIP as a template.K. The telomerase activity of liver cancer stem cells was detected by quantitative telomerase activity assay (TRAP). L. RNA pull down was detected by biotin-labeled TERC RNA probe (Biotin-TERT), and Western blotting was performed with anti-TERT, anti-HP1 $a$ and anti-P53, respectively. Western blotting of anti-Biotin was used as INPUT to add probe amount, and Western blotting of anti-HistoneH3 was performed as an internal reference of the reaction nuclear protein by using the protein extract before RNA pull down. M. RNA-immunoprecipitation (RIP) using anti-TERT, anti-P53, and anti-HP1a, and primers designed with TERC sequence were used for RT-PCR amplification.N. telomerase activity of liver cancer stem cells was examined by a method for quantitative telomerase activity assay (TRAP). 
A

a

D

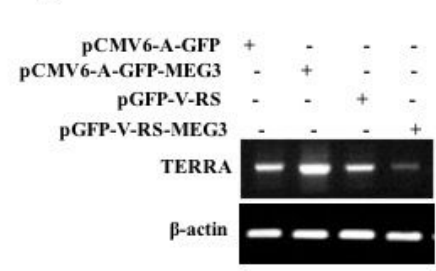

b

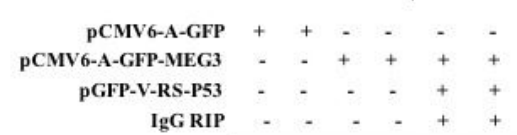

TERT RIP TERRART-PCR $-\quad \square$

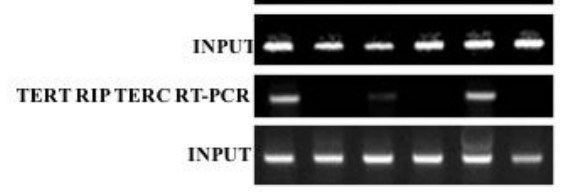

F

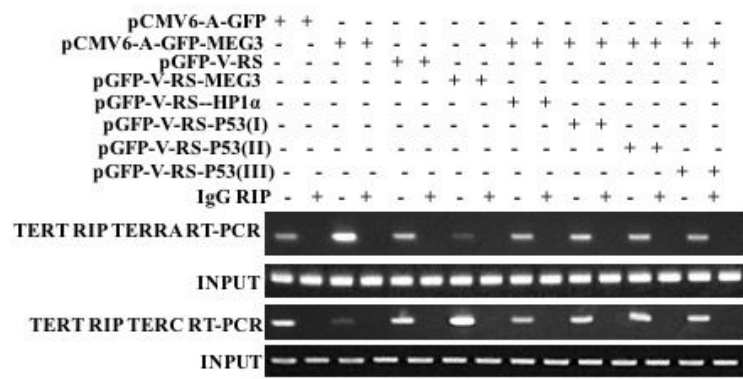

B

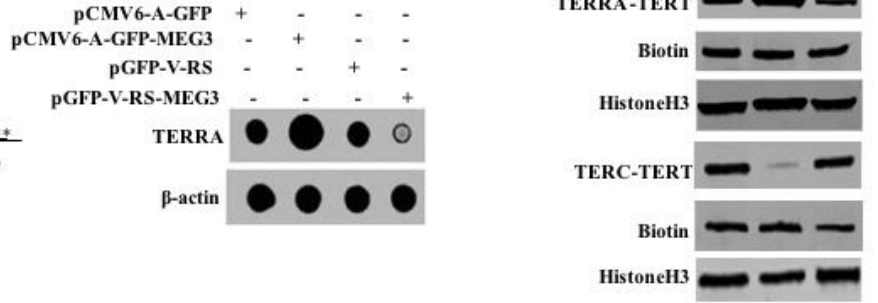

E

a

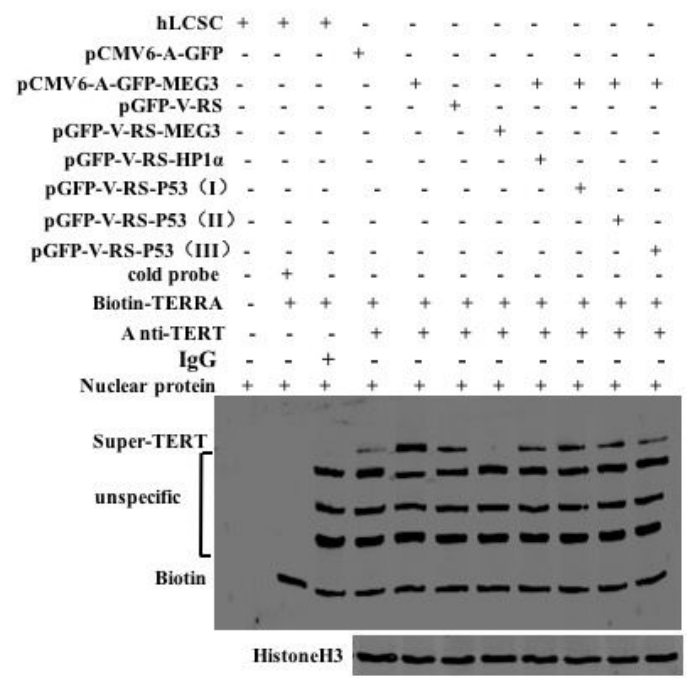

G

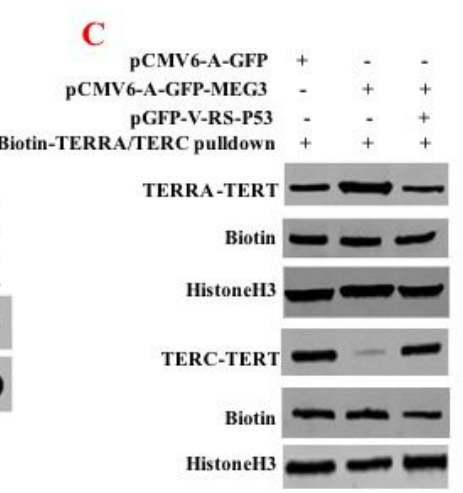

b
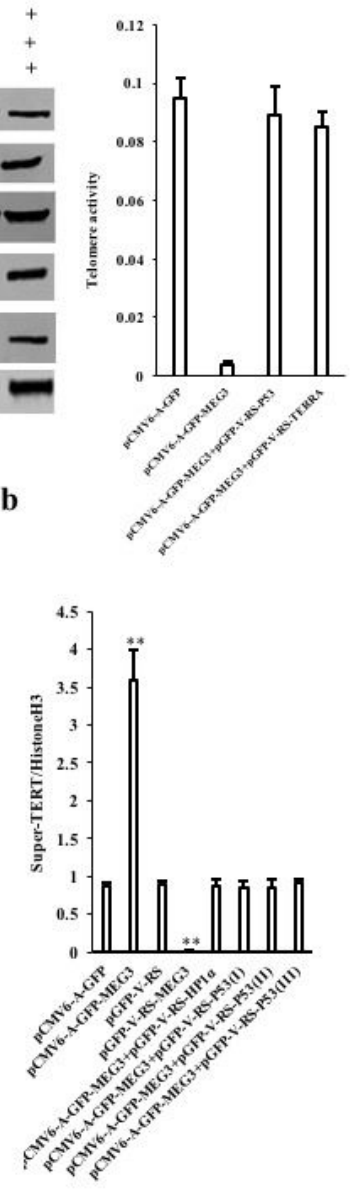

\section{Figure 5}

MEG3 inhibits telomerase activity in human liver cancer stem cells by long non-coding RNA TERRA. A. a. The transcriptional ability of TERRA in these four stable cell lines was detected by reverse transcription polymerase chain reaction (RT-PCR), and $\beta$-actin was used as Internal reference gene. b. Quantitative reverse transcription polymerase chain reaction (RT-PCR) was used to detect the expression of TERRA in these four stable cell lines, and $\beta$-actin was used as an internal reference gene. $B$. The transcriptional capacity of TERRA in these four stable cell lines was detected by Dot-blot, and GAPDH was used as an internal reference gene. C. RNA pulldown analysis was performed using TERRA or TERC biotin-labeled probes Biotin-TERRA or Biotin-TERC and anti-TERT. Hisone H3 is used as INPUT and Biotin is used as an internal reference.D. The RNA immunoprecipitation (RIP) with anti-P53 was used to extract TERRA or TERC by RT-PCR using primers designed by TERRA or TERC sequences using RNA isolated from RNA coprecipitation as a template. IgG RNA co-immunoprecipitation was used as a negative control, and TERRA or TERC was separately amplified as an internal reference (INPUT) using RNA retained before RIP as a template.E. Super-RNA-protein complex gel migration assay (Super-EMSA) using the biotin labeled TERRA RNA probe (Biotin-TERRA) and anti-TERT . IgG super-EMSA was used as a negative control, EMSA 
without nucleoprotein and EMSA with excess cold probe were used as a system reference, and the hybridization band of Biotin-TERRA and the amount of nucleoprotein added were used as INPUT. B. Quantitative analysis of gray scales of positive bands. F. After cross-linking formaldehyde, RNA antiprecipitation (RIP) using anti-TERT was performed. TERC and TERRA Primers were designed for RTPCR amplification. IgG RNA co-immunoprecipitation was used as a negative control, and TERC or TERRA was separately amplified as an internal reference (INPUT) using RNA retained before RIP as a template. G. the telomerase activity of liver cancer stem cells was examined by the quantitative telomerase activity assay (TRAP).

\section{Figure 6}

A

B

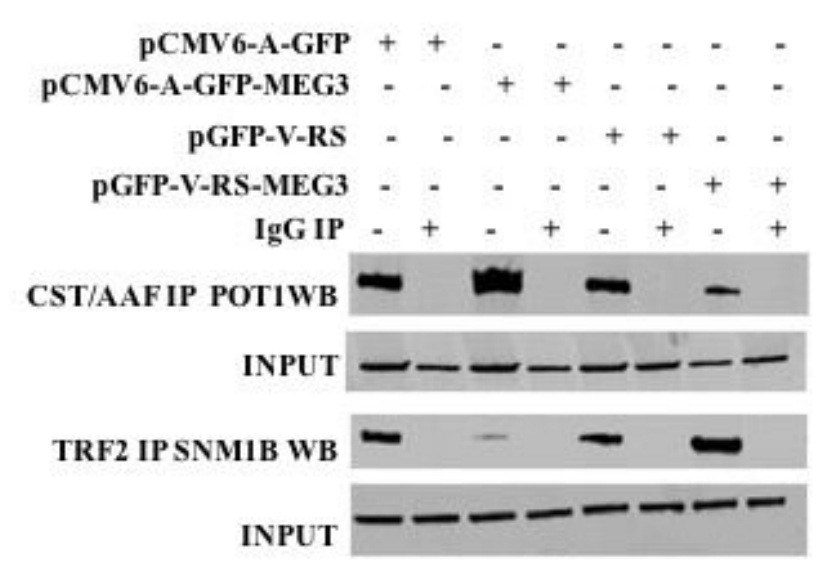

C

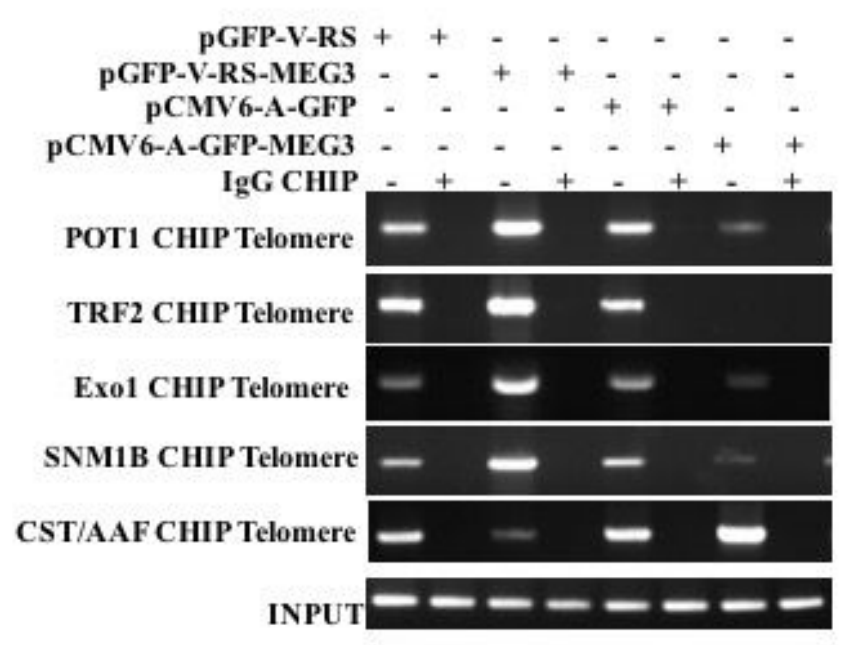

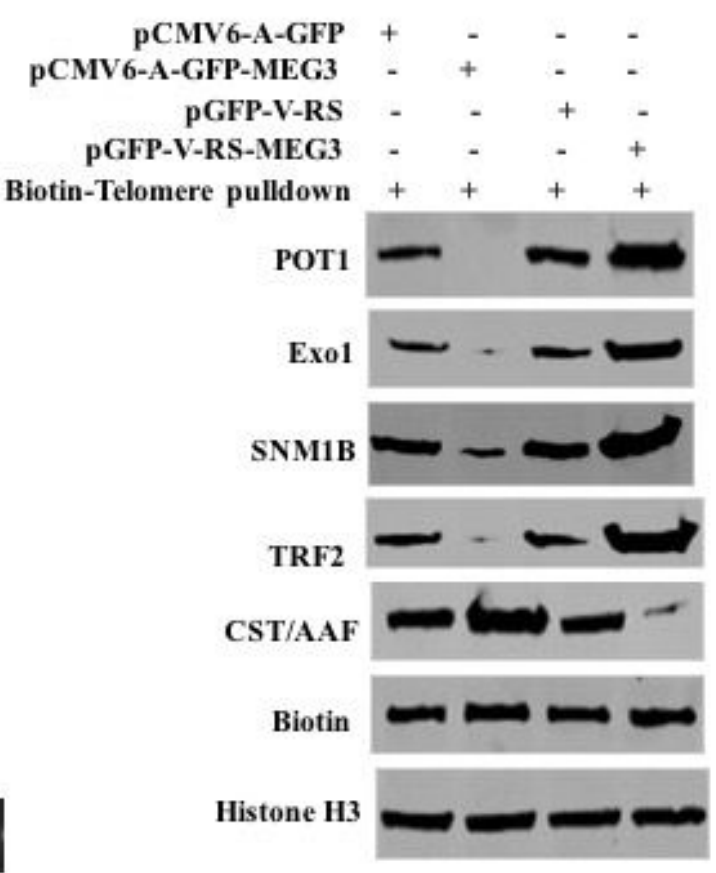

\section{Figure 6}

MEG3 inhibits the binding of POT1, Exol, TRF2, SNM1B to telomeric DNA and enhances the binding of CST/AAF to telomeric DNA in liver cancer stem cells. A. the co-immunoprecipitated with anti-CST/AAF or 
anti-TRF2. The precipitates were analyzed by Western blotting with anti-POT1 or anti-SNM1B. IgG coimmunoprecipitation was used as a negative control, and anti-CST/AAF or anti-TRF1 in the sample before coprecipitation was detected by Western blotting as INPUT.B. DNA pull down assay using biotinlabeled telomere DNA probe (Biotin-telomere DNA) with Western anti-POT1, anti-Exol, anti-TRF2, antiSNM1B and anti-CST/AAF Blotting detection analysis. Western blotting of anti-Biotin was used as an internal reference (INPUT) for the amount of probe added, and Western blotting of anti-Histone H3 was performed as an internal reference (INPUT).C. Analysis of chromatin immunoprecipitation (CHIP) using anti-POT1, anti-Exol, anti-TRF2, anti-SNM1B and anti-CST/AAF . The polymerase chain reaction (PCR) amplification was carried out using a primer designed according to the telomeric DNA. IgG chromatin immunoprecipitation was used as a negative control; telomere (using the same primers as above) was used as an internal reference (INPUT) by using the DNA retained before chromatin immunoprecipitation as a template.

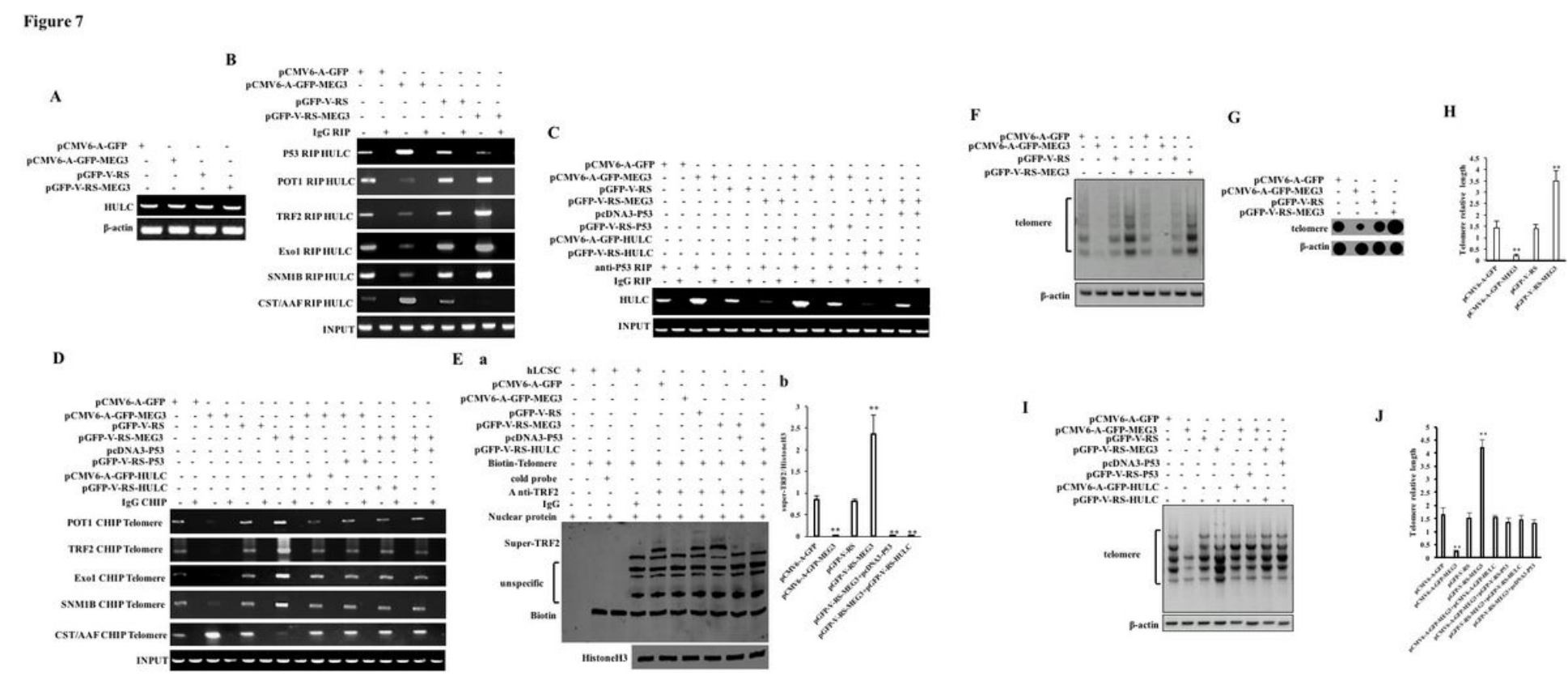

\section{Figure 7}

MEG3-dependent long non-coding RNA HULC and P53 affects the binding of POT1, Exol, TRF2, SNM1B, CST/AAF to telomeric DNA in human liver cancer stem cells. A. Reverse transcription- polymerase chain reaction (RT-PCR) to detect the transcriptional capacity of HULC in these four stable cell lines, $\beta$-actin as an internal reference gene. B. RNA immunoprecipitation (RIP) with anti-POT1, anti-Exol, anti-TRF2, antiSNM1B, anti-P53. RT-PCR amplification of HULC using primers designed by HULC sequence. IgG RNA RIP was used as a negative control, and HULC was separately amplified as an internal reference (INPUT) using RNA retained before RIP as a template.C. RNA immunoprecipitation (RIP) with anti-P53 and then amplification HULC by RT-PCR. IgG RNA immunoprecipitation was used as a negative control, and HULC was separately amplified as an internal reference (INPUT) using RNA retained before RIP as a template. D. chromatin immunoprecipitation (CHIP)) using anti-POT1, anti-Exol, anti-TRF2, anti-SNM1B, and antiCST/AAF. The DNA isolated and purified from the chromatin immunoprecipitate was used as a template, and a polymerase chain reaction (PCR) amplification was the telomeric DNA. IgG chromatin 
immunoprecipitation was used as a negative control.E. a. Both Biotin-labeled telomere probes (BiotinTelomere) and anti-TRF2, anti-Biotin were used for Super-RNA-protein complex gel migration experiments (Super-EMSA). IgG super-EMSA as a negative control. Nucleoprotein-free EMSA and EMSA with excess cold probe added as a system reference. Biotin-telomere hybridization band and the amount of nucleoprotein added as INPUT. b. Quantitative analysis of gray scales of positive bands.

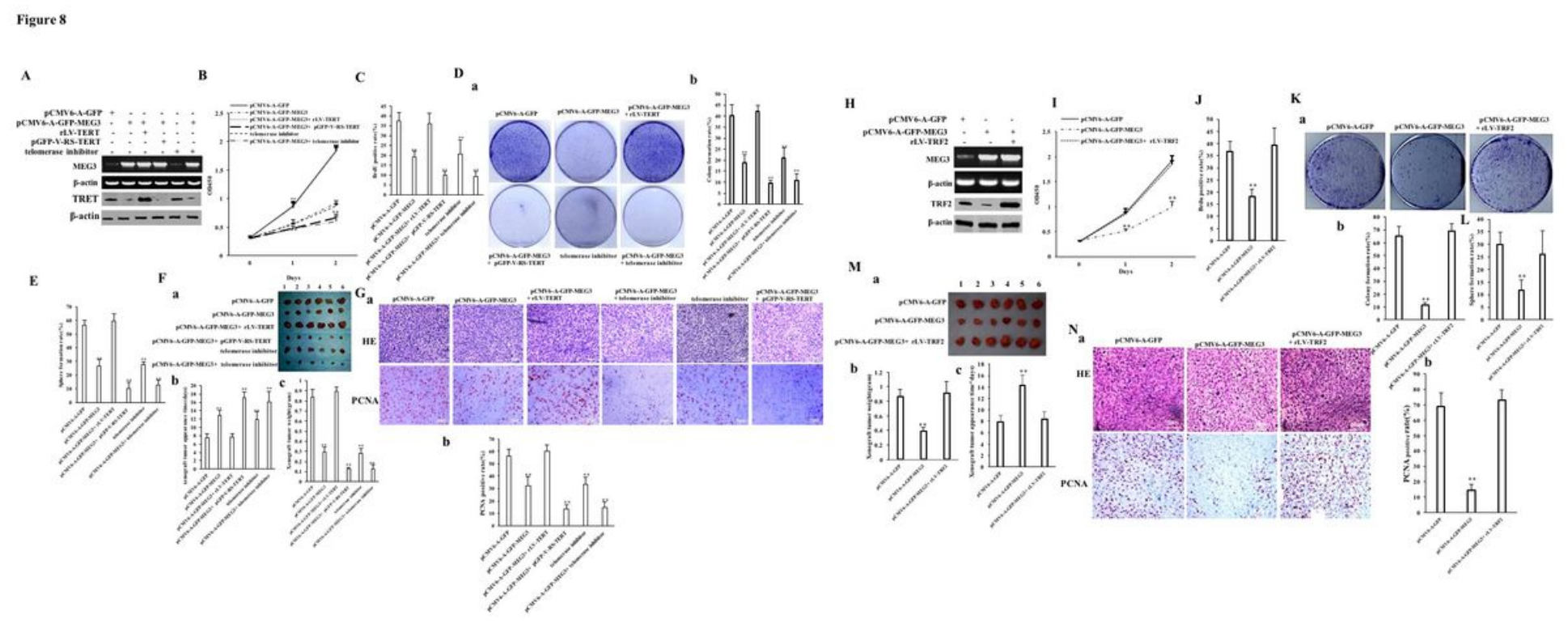

\section{Figure 8}

The Excessive of telomerase or TRF2abrogates the inhibitory effect of MEG3 on the growth of human liver cancer stem cells. A. MEG3 Transcriptional ability was by reverse polymerase chain reaction (RT$P C R), \beta$-actin as an internal reference gene. Total protein was extracted and the expression of TERT was detected by Western blotting, and $\beta$-actin was used as an internal reference gene. B. CCK8 assay for cell proliferation capacity.C. Determination of cell $S$ phase of these stably transfected cell lines by BrdU method percentage. D. Determination of colony forming ability a. Photograph of colonies; $b$. analysis of cell colony formation rate.E. The formation rate of sphere was measured. F. In vivo tumor formation test of liver cancer stem cells. a. Photographs of transplanted tumors (xenograft).b. Comparison of the size (g) of transplanted tumors in nude mice.c. comparison of time (days) of transplanted tumors in nude mice.G. a. $4 \%$ formalin-fixed, paraffin-embedded nude mouse transplanted tumor tissue sections $(4 \mu \mathrm{m})$ were subjected to HE staining and immunohistochemical staining for anti-PCNA. b. Comparison of PCNA positive rates of transplanted tumors in nude mice.H. MEG3 was detected by reverse transcription polymerase chain reaction (RT-PCR), and $\beta$-actin was used as an internal reference gene. Total cellular proteins were extracted, and the expression of TRF2 was detected by Western blotting, and $\beta$-actin was used as an internal reference gene.I. Cell proliferation ability was determined by CCK8 method..J. The percentage of cell S phase was determined by the BrdU staining. K. a. Photograph of plate colony; $b$. Cell plate colony formation rate analysis.L. The assay for sphere formation ability. M.a. photograph of a transplanted tumor (xenograft).b.The comparsion of xenpgraft tumors size (g). c. The comparison of xenograft tumor appearance time. N.a. HE staining and anti-PCNA immunohistochemical staining of $4 \%$ 
formalin-fixed, paraffin-embedded xenograft tumor tissue sections $(4 \mu \mathrm{m})$. b. Comparison of PCNA positive rates.

\section{Figure 9}

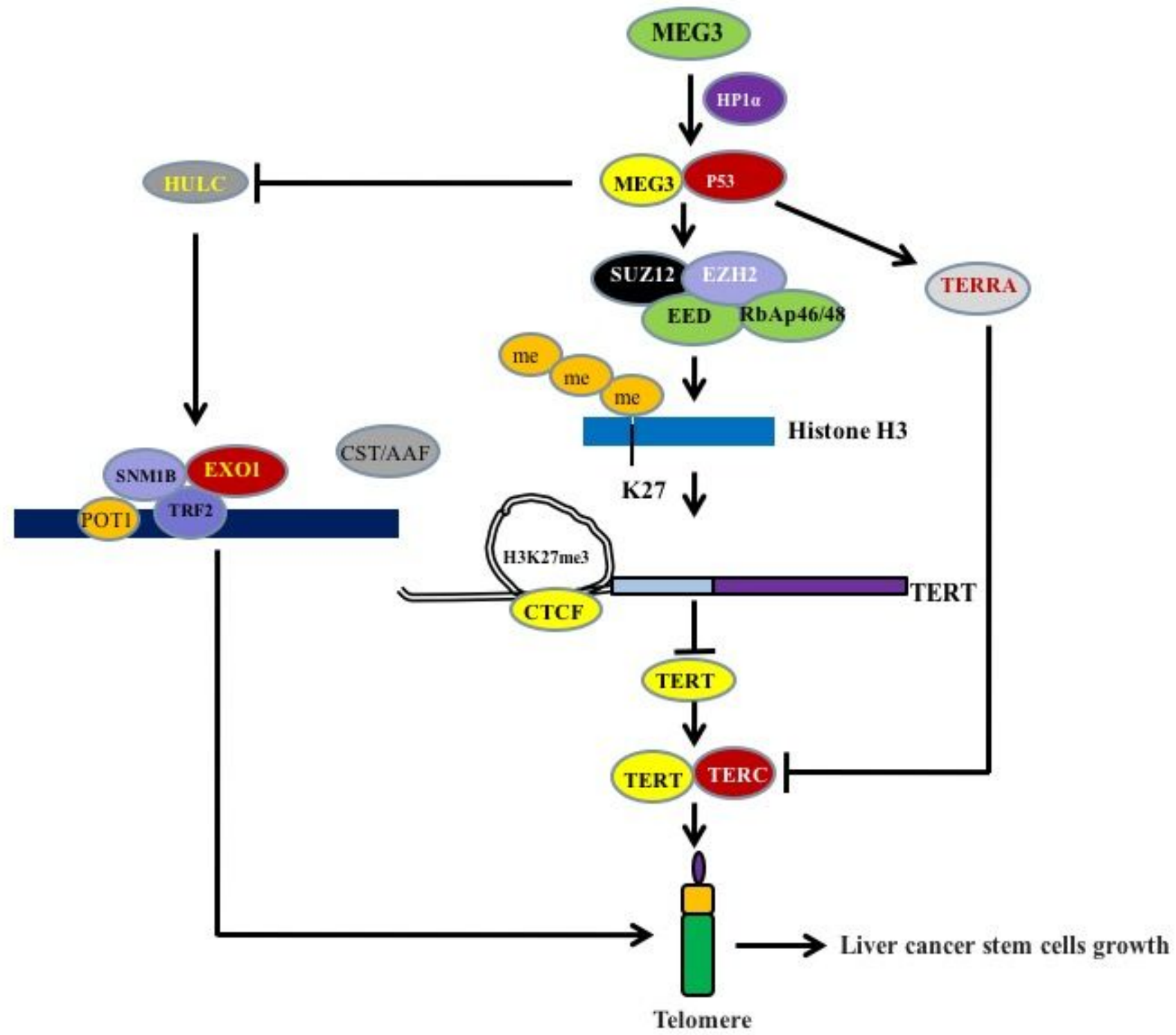

Figure 9

Schematic diagram of the molecular mechanism of MEG3 affecting the growth of human liver cancer stem cells

\section{Supplementary Files}

This is a list of supplementary files associated with this preprint. Click to download.

- supplementaldata.docx 